\title{
Biodiversidad de algas epizoicas en el Pacífico tropical mexicano
}

\author{
Biodiversity of epizoic algae in the Mexican tropical \\ Pacific
}

\section{Acta Botanica Mexicana}

\author{
Nataly Quiroz-González' (iD), Luis Gabriel Aguilar-Estrada² (iD, Ivette Ruiz-Boijseauneau² (D), Dení Rodríguez², (iD
}

\section{Resumen:}

Antecedentes y Objetivos: El conocimiento ficológico para la región del Pacífico mexicano es amplio, la mayoría de los estudios han estado orientados a conocer la flora adherida a sustratos rocosos. Sin embargo, es reducido el número de trabajos acerca de macroalgas que crecen sobre organismos vivos, como las algas epizoicas. El presente trabajo tiene como finalidad contribuir al conocimiento de la flora algal epizoica en el Pacífico tropical mexicano.

Métodos: Se llevaron a cabo cuatro muestreos del molusco Chiton articulatus en dos localidades de Guerrero, México, durante 2014, para conocer las algas que crecen sobre él. Además, se realizó una revisión bibliográfica de estudios en el Pacífico tropical mexicano para obtener los registros de las algas que crecen sobre animales. Finalmente, se determinaron las algas en los moluscos mediante observaciones de talos completos y cortes.

Resultados clave: En total se reportaron 124 especies, 81 géneros, 51 familias y 27 órdenes, distribuidos en cuatro divisiones de algas. Los órdenes con mayor riqueza específica fueron Ceramiales (23) y Corallinales (20) y las familias con mayor número de especies fueron Rhodomelaceae (13), Cladophoraceae (11) y Lithophyllaceae (10). Los géneros más diversos fueron Amphiroa y Cladophora (7). Se presentan, para Guerrero, 12 nuevos registros de especies, de los cuales tres son además nuevos registros para el Pacífico tropical mexicano y tres para el Pacífico de México. El estado con mayor número de especies registradas fue Guerrero (58). El sustrato más común fueron los moluscos. El grupo morfofuncional algal predominante fue los filamentos.

Conclusiones: Los resultados de este trabajo demuestran que la biodiversidad de algas presente en los sustratos animales es alta, y contribuye notablemente a la riqueza específica registrada para Guerrero y para el Pacífico de México. Se hace patente la importancia de desarrollar más estudios que se enfoquen al conocimiento de la epibiosis algal.

Palabras clave: epibiosis, ficoflora, Guerrero, quitones, riqueza específica.

\section{Abstract:}

Background and Aims: Although the phycological knowledge for the Mexican Pacific region is broad, most studies have been oriented to know the flora adhered to rocky substrates. However, the number of works on macroalgae that grow on living organisms, such as epizoics, is limited. The purpose of this paper is to contribute to the knowledge of epizoic algal flora in the Mexican tropical Pacific.

Methods: Four samplings of the mollusk Chiton articulatus were carried out in two locations in Guerrero, Mexico, during 2014, to know the algae that grow on it. Furthermore, a literature review of studies in the Mexican tropical Pacific was carried out to obtain the records of the algae that grow on animals. Finally, algae on molluscs were determined by observations of complete thallus and sections.

Key results: A total of 124 species, 81 genera, 51 families and 27 orders was reported, distributed in four algae divisions. The orders with the highest specific richness were Ceramiales (23) and Corallinales (20), and the families with the highest number of species were Rhodomelaceae (13), Cladophoraceae (11) and Lithophyllaceae (10). The most diverse genera were Amphiroa and Cladophora (7). For Guerrero, 12 new species records are presented, of which three are also new records for the Mexican tropical Pacific and three for the Mexican Pacific. The state with the highest number of recorded species was Guerrero (58). The most common substrate was mollusks. The predominant algal morphofunctional group were the filaments.

Conclusions: The results of this work demonstrate that the biodiversity of algae present on animal substrates is high, contributing significantly to the specific richness recorded for Guerrero and the Mexican Pacific. The importance of developing more studies that focus on knowledge of algal epibiosis is evident.

Key words: chitons, epibiosis, ficoflora, Guerrero, specific richness.

${ }^{1}$ Universidad Nacional Autónoma de México, Programa de posgrado, Instituto de Ciencias del Mar y Limnología, Av. Universidad 3000, Circuito Exterior s.n., Ciudad Universitaria, Coyoacán, 04510 Cd. Mx., México.

${ }^{2}$ Universidad Nacional Autónoma de México, Facultad de Ciencias, Av. Universidad 3000, Circuito Exterior s.n., Ciudad Universitaria, Coyoacán, 04510 Cd. Mx., México.

${ }^{3}$ Autor para la correspondencia: denirodriguez@ciencias.unam.mx
Recibido: 5 de diciembre de 2019.

Revisado: 7 de enero de 2020.

Aceptado por Marie-Stéphanie Samain: 24 de marzo de 2020.

Publicado Primero en línea: 26 de mayo de 2020.

Publicado: Acta Botanica Mexicana 127 (2020).
Citar como: Quiroz-Gónzalez, N., L. G. Aguilar-Estrada, I. Ruiz-Boijseauneau y D. Rodríguez. 2020. Biodiversidad de algas epizoicas en el Pacífico tropical mexicano. Acta Botanica Mexicana 127: e1645. DOI: 10.21829/ abm127.2020.1645 


\section{Introducción}

Las macroalgas bentónicas se desarrollan adheridas a un sustrato con el cual no mantienen ningún tipo de relación trófica (Báez y Flores-Moya, 2003) y se denominan epizoicas a las que para desarrollarse usan como basibionte a algún animal (Wahl, 2009). A nivel mundial existen pocos estudios que analizan la epibiosis de algas en animales; la mayoría se refieren a tortugas y peces basibiontes y en menor medida a invertebrados como moluscos, corales y esponjas sobre los que se han encontrado las siguientes especies de algas: Aglaothamnion boergesenii (Aponte \& D.L. Ballantine) L' Hardy-Halos \& Rueness, Blidingia marginata (J. Agardh) P.J.L. Dangeard ex Bliding, Centroceras clavulatum (C. Agardh) Montagne, Chaetomorpha antennina (Bory) Kützing, C. linum (O.F. Müller) Kützing, Champia parvula (C. Agardh) Harvey, Cladophora albida (Nees) Kützing, C. vagabunda (L.) Hoek, Ectocarpus siliculosus (Dillwyn) Lyngbye, Sphacelaria tribuloides Meneghini, Scytonematopsis crustacea (Thuret ex Bornet \& Flahault) Koválik \& Komárek (Frazier et al., 1985; Bretos y Chihuailaf, 1990; BaIlantine et al., 2001; Báez et al., 2001, 2002; Báez y FloresMoya, 2003; Connelly y Turner, 2009; Levenets et al., 2010; Serio et al., 2011; Martins et al., 2014).

Aunque el conocimiento ficológico para la región del Pacífico mexicano es amplio, la mayoría de los estudios han estado orientados a conocer la flora local que se encuentra adherida a sustratos rocosos. Es menor el número de trabajos desarrollados para conocer las macroalgas que crecen sobre organismos vivos, ya sea como epífitos o como epizoicos (González-González et al., 1996; Mateo-Cid y Mendoza-González, 1991, 1992, 2012; Mendoza-González y Mateo-Cid, 1985, 1986, 1998; Pedroche y Sentíes, 2003a; Dreckmann et al., 2006; Vázquez-Delfín, 2008; Vega et al., 2008; Mendoza-González et al., 2011).

Solo tres estudios se han desarrollado específicamente sobre algas epizoicas marinas, uno para el Caribe mexicano acerca de especies que crecen sobre tortugas marinas (Sentíes et al., 1999), en el que se registraron 37 taxa de algas epizoicas, destacando los órdenes Ceramiales y Cladophorales; otro para Jalisco con epibiontes de tortugas golfinas que incluyó especies del género Polysiphonia Greville (Hernández-Vázquez y Valadez-González, 1998) y recientemente, Álvarez-Cerrillo et al. (2017) reportaron los epibiontes de Chiton articulatus G.B. Sowerby I 1832, capturados en las costas de Guerrero, a partir de la presencia de dos grupos morfofuncionales, filamento y costra, pero no determinaron categorías taxonómicas.

El objetivo de este trabajo es hacer un aporte al conocimiento de la ficoflora epizoica registrada para el Pacífico tropical mexicano. Por un lado, el estudio se basó en las algas epizoicas en la cucaracha de mar Chiton articulatus (Mollusca, Polyplacophora) en la costa del estado de Guerrero, México, y por otro lado, en la revisión de la literatura publicada entre 1944 y 2019 sobre este tema.

\section{Materiales y Métodos}

\section{Captura de organismos}

En esta contribución se da información de las especies de macroalgas que crecen sobre las conchas del molusco Chiton articulatus y que fueron capturados por los autores en la región de Ixtapa-Zihuatanejo, la cual se localiza en la porción suroeste de México, en las costas del estado de Guerrero. En enero, mayo, julio y noviembre de 2014, los animales se capturaron de forma manual con ayuda de una espátula en dos localidades: 37 individuos en el intermareal rocoso medio y alto de playa El Palmar $\left(17^{\circ} 39^{\prime} 0.4^{\prime \prime} \mathrm{N}\right.$, $\left.101^{\circ} 36^{\prime} 2.79^{\prime \prime O}\right)$, en el extremo norte de Ixtapa y 25 en el intermareal rocoso del muelle municipal $\left(17^{\circ} 38^{\prime} 13.88^{\prime \prime} \mathrm{N}\right.$, $101^{\circ} 33^{\prime} 31.87^{\prime \prime O}$ ), en la bahía de Zihuatanejo (Fig. 1).

Los organismos se colocaron en bolsas con agua de mar para ser transportados al laboratorio de la Unidad Multidisciplinaria de Docencia e Investigación Zihuatanejo (UMDI-Z) de la Universidad Nacional Autónoma de México (UNAM), se preservaron en frío a $4{ }^{\circ} \mathrm{C}$ para su relajación y a continuación fueron colocados en formol para su posterior identificación.

Los organismos se identificaron con base en sus caracteres conquiliológicos (caracteres morfológicos de la concha) y con apoyo de la literatura malacológica especializada para el Océano Pacífico (Keen, 1971; Kaas et al., 2006). Los ejemplares fueron depositados en la colección, en proceso de registro ante SEMARNAT, "Invertebrados Asociados a Macroalgas" del laboratorio de Ficología marina (Biodiversidad Marina), UNAM.

De 62 individuos capturados de Chiton articulatus, se revisaron 26 (16 para playa El Palmar y 10 para el muelle 


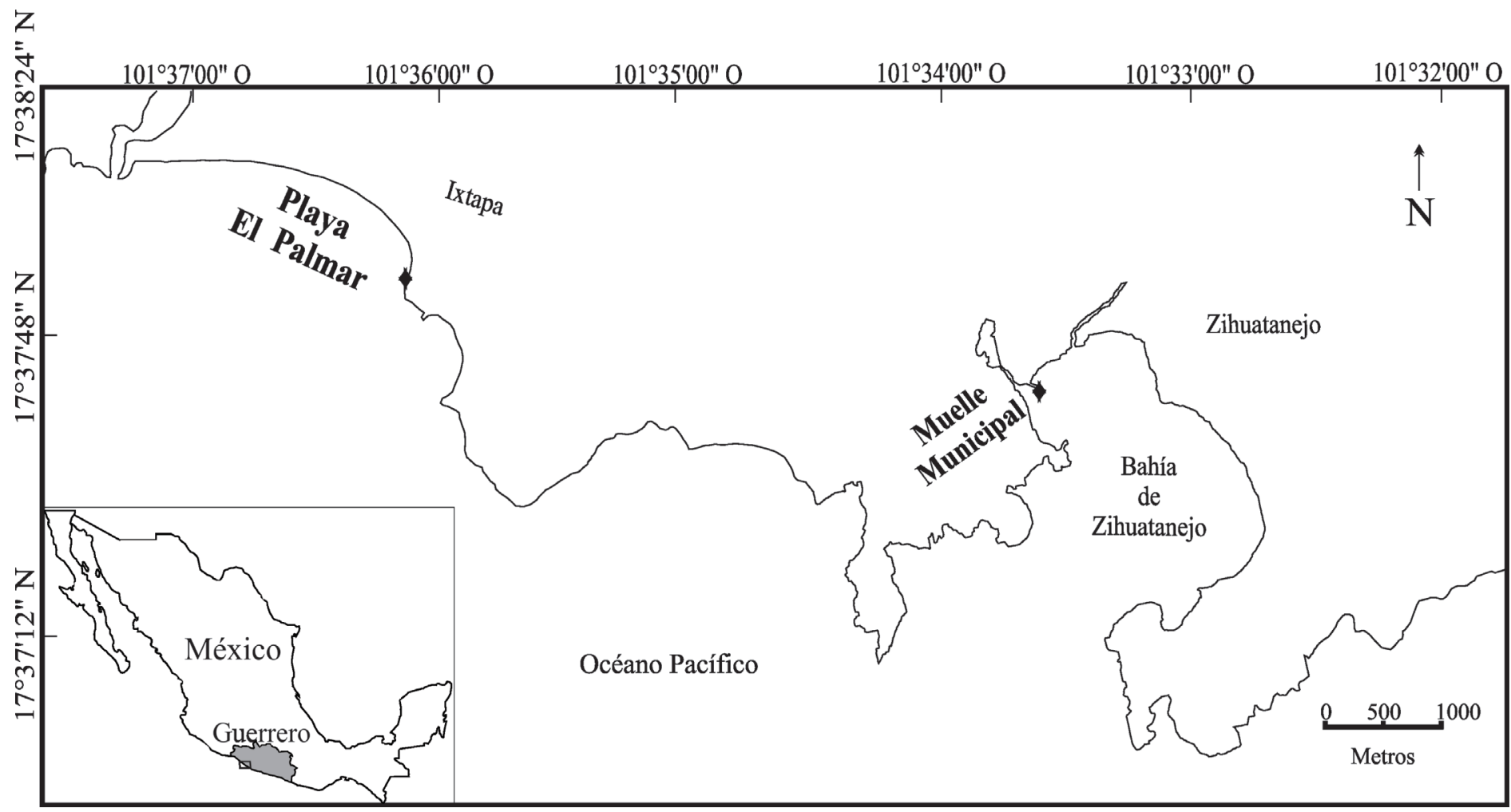

Figura 1: Área de estudio, Ixtapa-Zihuatanejo, Guerrero, México.

municipal) para la identificación de las algas, ya que sólo en esos se encontraron creciendo dichos organismos.

\section{Identificación de algas}

A partir de la observación de las características morfológicas de las algas con apoyo de microscopía estereoscópica (Nikon CLEDS, Tokio, Japón) y de preparaciones semipermanentes en gelatina glicerinada de los talos y de los cortes transversales observadas con microscopía óptica (OLYMPUS-CX23, Tokio, Japón), se realizó la determinación taxonómica de las algas empleando la siguiente literatura ficológica especializada: Taylor (1945, 1960), Dawson (1953a, b, 1954, 1960, 1961a, b, 1962, 1963a, b), Abbott y Hollenberg (1976) y Anagnostidis y Komárek (1988).

\section{Revisión bibliográfica}

Se revisaron 80 trabajos realizados entre 1944-2019, dados a conocer en artículos científicos, capítulos de libro y tesis de licenciatura y posgrado, que incluían información de ocho estados (Sinaloa, Nayarit, Jalisco, Colima, Michoacán, Guerrero, Oaxaca y Chiapas) a lo largo de la costa del
Pacífico tropical mexicano (PTM). Dichos documentos se obtuvieron de repositorios en línea, de bases de datos y de la biblioteca del laboratorio de Ficología (Biodiversidad Marina) de la Facultad de Ciencias, UNAM. Se seleccionaron aquellos trabajos florísticos y ecológicos realizados en localidades del PTM desde el trabajo más antiguo realizado en estas costas hasta los estudios más recientes, se revisaron los resultados, las listas de especies y las descripciones de dichas investigaciones en búsqueda de los sustratos; en el caso de tratarse de un animal, se realizó su registro junto con la especie de alga epizoica. En el presente estudio se incluyen y se citan todos los documentos que presentan información al respecto.

Con los datos provenientes de la revisión de las algas que crecen en las conchas de Chiton articulatus capturados en la región de Ixtapa-Zihuatanejo y de los registros de la literatura, se elaboró una lista de especies, arreglada sistemáticamente y actualizada con AlgaeBase (Guiry y Guiry, 2020). Para los nuevos registros se llevó a cabo una descripción morfológica. Se registraron los grupos morfofuncionales a los que cada especie pertenece (microscópicas, 
filamento, folioso, foliosas corticadas, filamento corticado, coriáceas, calcáreas articuladas, costras), de acuerdo con la clasificación de Steneck y Dethier (1994). Cada especie de algas se asoció a un tipo de estructura de fijación al sustrato.

\section{Resultados}

\section{Inventario de algas epizoicas en Chiton articulatus}

En total se capturaron 62 ejemplares de quitones en la zona de Ixtapa-Zihuatanejo, Guerrero, México. De éstos, 26 presentaron algas epibiontes (42\%). Se registraron 35 especies de algas pertenecientes a cuatro divisiones, 16 especies de Chlorophyta, 15 de Rhodophyta, dos de Ochrophyta-Phaeophyceae y dos de Cyanobacteria. De éstas, 12 son nuevos registros para la flora de macroalgas del estado de Guerrero, de las cuáles seis son exclusivamente para Guerrero. Del resto, tres especies representan nuevos registros para el Pacífico tropical mexicano y tres lo son para el Pacífico de México. De la revisión bibliográfica solo se encontraron registros de algas epizoicas en 18 estudios que incluyen información de ocho estados a lo largo de la costa del Pacífico tropical mexicano (Apéndice).

En total se registraron 124 especies de algas al integrar los reportes de las que crecen sobre las conchas de Chiton articulatus y de la revisión bibliográfica (Apéndice), las cuales están distribuidas en 81 géneros, 51 familias, 27 órdenes y cuatro divisiones, incluyendo 79 especies de Rhodophyta, 27 de Chlorophyta, 13 de OchrophytaPhaeophyceae y cinco de Cyanobacteria. Adicionalmente, 12 ejemplares solo pudieron ser identificados hasta nivel de género, debido al estado de preservación de los talos o a la cantidad de estos, que imposibilitó la observación de caracteres diagnósticos a nivel de especie; estos no se incluyeron en los conteos del presente trabajo. Los órdenes mejor representados fueron Ceramiales con 23 especies y Corallinales con 20. Las familias con mayor riqueza de especies fueron Rhodomelaceae (13), Cladophoraceae (11) y Lithophyllaceae (10), mientras que los géneros mejor representados fueron Amphiroa y Cladophora, con siete especies cada uno.

\section{Riqueza por estado}

Guerrero es el estado con el mayor número de algas registradas como epizoicas (58 especies), seguido de Nayarit
(21) y Sinaloa (11), mientras que en Chiapas únicamente se ha registrado una. Quince especies se compartieron entre los distintos estados, las del género Gelidium J.V. Lamouroux se reportan para siete estados y en particular $G$. pusillum (Stackhouse) Le Jolis ha sido registrada en cinco estados, así como Ulva intestinalis L. en cuatro (Apéndice).

\section{Grupos morfofuncionales}

El grupo predominante fue el de los filamentos con 57 especies, seguido de los filamentos corticados (27) y de las algas costrosas (20) (Apéndice).

\section{Tipos de basibiontes}

De acuerdo con los resultados de las recolecciones realizadas en este estudio de algas epizoicas de Chiton articulatus y con los reportes de la información bibliográfica revisada, se identificaron cuatro basibiontes animales: balanos, coral vivo, esponjas y moluscos. Estos últimos presentaron el mayor número de epibiontes (66 especies), seguidos de los corales vivos (23), esponjas (seis) y balanos (una). Por su parte, 13 especies de algas se encontraron creciendo en más de un basibionte (Amphiroa beauvoisii J. V. Lamouroux, A. misakiensis Yendo, Caulerpa cupressoides (Vahl) C. Agardh, C. racemosa (Forsskål) J. Agardh, C. sertularioides (S.G.Gmelin) M. Howe, Cladophora microcladioides Collins, Crusticorallina muricata (Foslie) P.W. Gabrielson, Martone, K.R. Hind \& C.P. Jensen, Derbesia marina (Lyngbye) Solier, Gelidium mcnabbianum (E.Y. Dawson) B. Santelices, G. microdentatum E.Y. Dawson, G. pusillum (Stackhouse) Le Jolis, Hildenbrandia rubra (Sommerfelt) Meneghini, Hypnea johnstonii Setchell \& N.L. Gardner). Gelidium pusillum (Stackhouse) Le Jolis fue el alga que se encontró en mayor número de basibiontes (moluscos, corales, esponjas y balanos). Para 14 especies de algas, aunque se menciona su condición de epizoicas, no se registra el basibionte donde se encontraron (Apéndice).

\section{Estructuras de fijación}

Los resultados de este trabajo muestran que la biodiversidad de algas presente en los sustratos animales es alta. Además, contribuye notablemente a la riqueza específica registrada para Guerrero y para el Pacífico de México. Se hace patente la importancia de desarrollar más estudios 
que se enfoquen al conocimiento de la epibiosis algal, además de la perspectiva florística, desde el punto de vista de las interacciones ecológicas.

El tipo predominante de forma de adhesión al basibionte fue el de los rizoides que presentaron 76 especies, seguido de los discos basales con 24 (Apéndice). Cabe destacar que 15 especies no presentan ningún tipo de estructura especializada; sin embargo, se sabe que estas algas segregan sustancias cementantes para la fijación al sustrato (algas costrosas). Por último, cinco especies presentaron mucílago (Cyanobacteria) y cuatro utilizan una célula basal como estructura de adhesión al sustrato.

\section{Descripción de nuevos registros}

A continuación, se presentan las descripciones de los 12 nuevos registros para Guerrero, de los cuales seis fueron exclusivos para dicho estado (indicados con *); además, tres representan nuevos registros para el Pacífico tropical mexicano $\left({ }^{*}\right)$, mientras que tres especies representan nuevos registros para el Pacífico de México $(* * *)$. Los talos de algunos de los especímenes descritos se observan en la figura 2.

\section{CHLOROPHYTA}

\section{Cladophorales}

\section{Cladophoraceae}

*Cladophora laetevirens (Dillwyn) Kützing. Phycologia generalis order Anatomie, Physiologie und Systemkunde der Tange 2: 263. 1843. TIPO: INGLATERRA. Gales, Swansea, Glamorgan, sin colector s.n (holotipo: no localizado).

Talo $1 \mathrm{~mm}$ de largo; células apicales 25-3 $\mu \mathrm{m}$ de diámetro, 130-225 $\mu \mathrm{m}$ de longitud, células en las porciones medias 30-50 $\mu \mathrm{m}$ de diámetro, 4-7 diámetros de longitud, hacia la base células 25-40 $\mu \mathrm{m}$ de diámetro, 6-10 diámetros de longitud; ápices redondeados y ligeramente curvados hacia la derecha; ramificación dicotómica hacia la base, se torna unilateral desde la zona media hacia el ápice, de primer o segundo orden de ramificación.
Distribución: Península de Baja California (León Tejera et al., 1993; Serviere-Zaragoza et al., 1993, 1998); Jalisco y Nayarit (León Tejera et al., 1993; Serviere-Zaragoza et al., 1993, 1998).

Hábitat: creciendo en valvas de Chiton articulatus.

Ejemplar examinado: MÉXICO. Guerrero, municipio Zihuatanejo de Azueta, playa El Palmar, 24.VII.2014, L. Aguilar et al. INV-835 (Invertebrados Asociados a Macroalgas, UNAM).

**Cladophora graminea Collins, Rhodora 11: 19. Fig. 6. 1909. TIPO: ESTADOS UNIDOS DE AMÉRICA. California, Monterey Península, A. E. Bush s.n. (holotipo: no localizado).

Talo en mechones hemisféricos, $0.5-1 \mathrm{~cm}$ de largo; células apicales 100-500 $\mu \mathrm{m}$ de diámetro; células basales 300-500 $\mu \mathrm{m}$ de diámetro, 20-30 veces más largas; ramas rígidas, en su mayoría largas e ininterrumpidas por ramificaciones a cierta distancia; ramificación dicotómica o tricotómica hacia la base, alterna arriba; ramitas con 1-3 células, estrechándose desde la base hasta el ápice.

Distribución: Baja California, Sonora (Dawson, 1944, 1945, 1949, 1951, 1953b, 1960, 1961b, 1966; Norris, 1975; Huerta, 1978; Littler y Littler, 1981; Aguilar y Bertsh, 1983; Aguilar y Pacheco, 1986; Aguilar et al., 1990).

Hábitat: creciendo en valvas de Chiton articulatus.

Ejemplar examinado: MÉXICO. Guerrero, municipio Zihuatanejo de Azueta, muelle municipal, 26.VII.2014, L. Aguilar et al. INV-825 (Invertebrados Asociados a Macroalgas, UNAM).

*Chaetomorpha californica Collins, Phycotheca borealiamericana. A collection of dried specimens of the algae of North America 664. 1900. TIPO: ESTADOS UNIDOS DE AMÉRICA. La Jolla, California, sin colector s.n. (holotipo: $N Y !)$. 


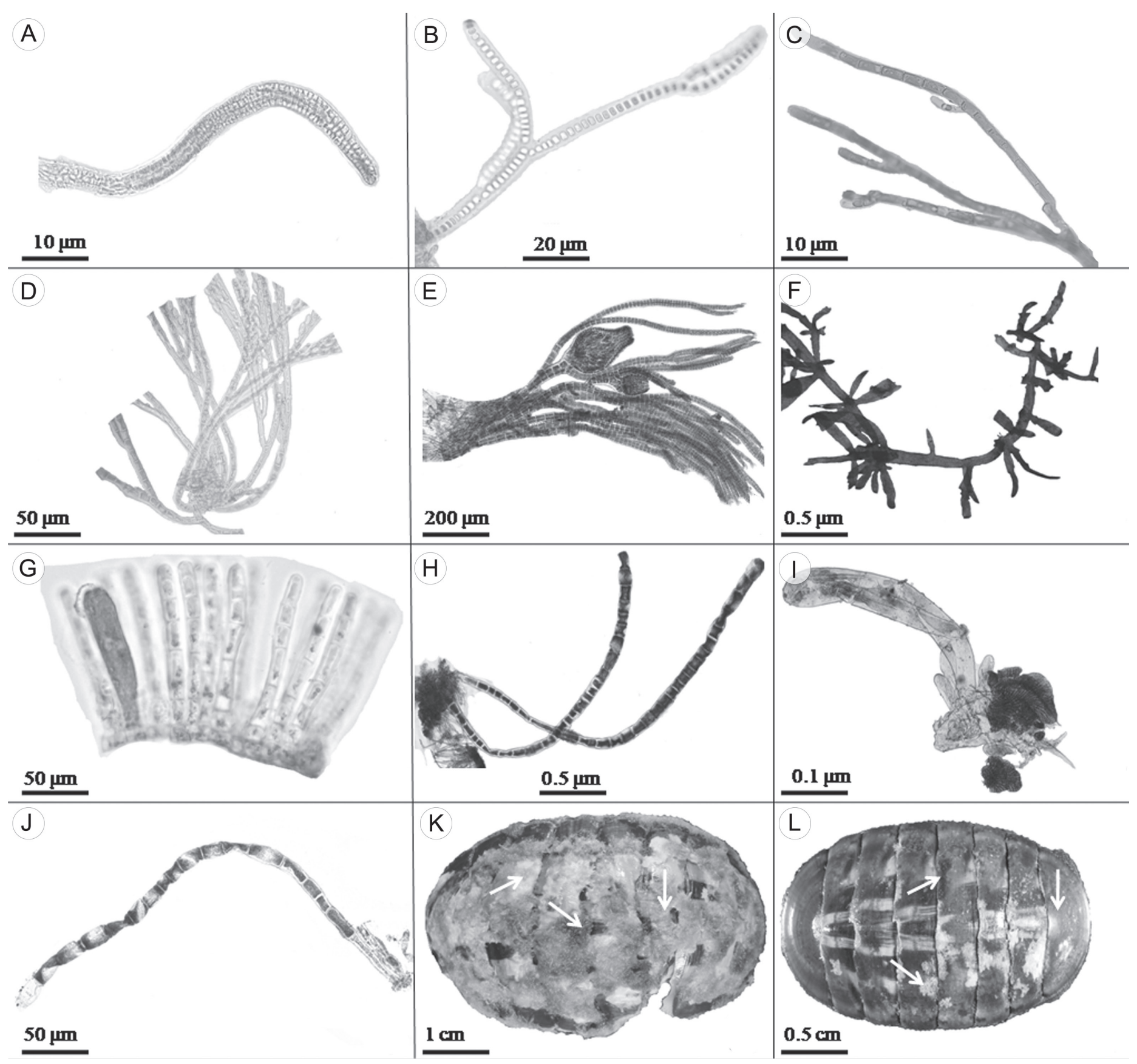

Figura 2: A. Porphyrostromium pulvinatum West \& Zuccarello; B. Chroodactylon ornatum (C. Agardh) Basson, filamentos ramificados; C. Colaconema coccineum (K.M. Drew) P.W. Gabrielson; D. Grania pectinata (Kylin) Athanasiadis; E. Polysiphonia pacifica Hollenberg, filamentos con cistocarpos; F. Gelidium pusillum (Stackhouse) Le Jolis; G. Myrionema strangulans Greville; H. Chaetomorpha linum (O.F. Müller) Kützing; I. Siphonocladus sp.; J. Ulothrix flacca (Dillwyn) Thuret; K, L. Chiton articulatus G.B. Sowerby, con algas epibiontes.

Talos filamentosos, anclados en el extremo proximal por medio de ramas rizoides no separadas que forman un disco; células apicales de diámetro uniforme, 20-25 $\mu \mathrm{m}$ de diámetro, 1-5 veces más largas que anchas; célula basal alargada 100-150 $\mu \mathrm{m}$ de largo; paredes laterales claramente engrosadas en capas.
Distribución: Michoacán, Oaxaca (Dreckmann et al., 1990; León-Tejera et al., 1993).

Hábitat: creciendo en valvas de Chiton articulatus. 
Ejemplar examinado: MÉXICO. Guerrero, municipio Zihuatanejo de Azueta, muelle municipal, 01.V.2014, L. Aguilar et al. INV-803 (Invertebrados Asociados a Macroalgas, UNAM).

*Chaetomorpha linum (O.F. Müller) Kützing, Phycologia germanica 204. 1845. TIPO: DINAMARCA. Nakskov Fjord, Lolland, sin colector s.n. (holotipo: no localizado). Fig. 2.

Talos filamentosos, erectos, uniseriados, anclados por medio de ramas rizoidales; células apicales de diámetro uniforme, 150-300 $\mu \mathrm{m}$ de diámetro, 1-2 veces más largas que anchas; células cercanas a la base 1-2.5 veces más largas que anchas; célula basal 750-1200 $\mu \mathrm{m}$ de largo.

Distribución: Colima, Jalisco, Nayarit, Oaxaca, Sinaloa y Sonora (Mendoza-González y Mateo-Cid, 1986; Ortega et al., 1987; Mateo-Cid y Mendoza-González, 1992; LeónTejera y González-González, 1993; Serviere-Zaragoza et al., 1993, 1998; Mendoza-González et al., 1994; Dreckmann y Gamboa Contreras, 1998; Aguilar et al., 2002).

Hábitat: creciendo en valvas de Chiton articulatus.

Ejemplares examinados: MÉXICO. Guerrero, municipio Zihuatanejo de Azueta, playa El Palmar, muelle municipal, 01-02.V.2014, L. Aguilar et al. INV-803, INV-805, INV816 (Invertebrados Asociados a Macroalgas, UNAM).

***Chaetomorpha nodosa Kützing, Species algarum 376. 1849. TIPO: GUYANA FRANCESA. Cayenne, Le Prieur s.n. (holotipo: NY!).

Talos filamentosos, aislados o formando grupos de dos a tres individuos, anclados al sustrato por una célula basal recta con base discoide; células apicales redondeadas; célula basal cónica, más larga que ancha, 20-25 $\mu \mathrm{m}$ de diámetro, 2.5-6 diámetros de longitud; el resto de las células con paredes lameladas y leves constricciones, 31-41 $\mu \mathrm{m}$ de diámetro, 0.6-1 diámetros de longitud.

Distribución: Atlántico mexicano (Quiroz et al., 2017).
Hábitat: creciendo en valvas de Chiton articulatus.

Ejemplar examinado: MÉXICO. Guerrero, municipio Zihuatanejo de Azueta, playa El Palmar, 02.V.2014, L. Aguilar et al. INV-816 (Invertebrados Asociados a Macroalgas, UNAM).

Ulotrichales

\section{Ulotrichaceae}

*Ulothrix flacca (Dillwyn) Thuret, Mémoires de la Société Impériale des Sciences Naturelles de Cherbourg 10: 156. 1863. TIPO: INGLATERRA. Gales, Swansea, Glamorgan, sin colector s.n. (lectotipos: BM!, K!). Fig. 2.

Talo filamentoso, filamentos uniseriados sin ramificaciones con múltiples septos, 23-39 $\mu \mathrm{m}$ de diámetro; células 10-37 $\mu \mathrm{m}$ de diámetro con paredes anchas, 6-7 $\mu \mathrm{m}$ de ancho, 28-45 $\mu \mathrm{m}$ de largo; cloroplastos en forma de anillo y con disposición parietal en las células; pirenoides 1-2.

Distribución: Michoacán (Stout y Dreckmann, 1993).

Hábitat: creciendo en valvas de Chiton articulatus.

Ejemplar examinado: MÉXICO. Guerrero, municipio Zihuatanejo de Azueta, playa El Palmar 02.V.2014, L. Aguilar et al. INV-816 (Invertebrados Asociados a Macroalgas, UNAM).

***Urospora penicilliformis (Roth) Areschoug, Nova Acta Regiae Societatis Scientiarum Upsaliensis, Ser. 3 6(2): 16. 1866. TIPO: ALEMANIA. Niedersachsen, Eckwarden, sin colector s.n. (holotipo: no localizado).

Filamentos unidos por rizoides retenidos dentro de las paredes laterales de las células; células multinucleadas, cuadradas a cilíndricas, 38-45 $\mu \mathrm{m}$ de diámetro, de diámetro uniforme; células, 0.5 - 1.5 veces más largas que anchas; con cloroplastos reticulados.

Distribución: desde Alaska al sur de California (Abbott y Hollenberg, 1976). 
Hábitat: creciendo en valvas de Chiton articulatus.

Ejemplar examinado: MÉXICO. Guerrero, municipio Zihuatanejo de Azueta, playa El Palmar 02.V.2014, L. Aguilar et al. INV-816 (Invertebrados Asociados a Macroalgas, UNAM).

**Urospora wormskioldii (Mertens ex Hornemann) Rosenvinge, Grønlands Havalger, Meddelelser om Grønland 3: 763-981. 1893. TIPO: DINAMARCA. Groenlandia, Ad littora Gothaab, sin colector s.n. (holotipo: no localizado).

Filamentos unidos por rizoides retenidos dentro de las paredes laterales de las células; células multinucleadas, cilíndricas o ligeramente constreñidas, 25-70 $\mu \mathrm{m}$ de diámetro, aumentan gradualmente su diámetro en forma distal a 120-300 $\mu \mathrm{m}$ en porciones terminales, cuadradas a alargadas, hasta 10 veces más largas que anchas; cloroplastos variables que van desde reticulados hasta en forma de banda.

Distribución: Baja California (Pedroche et al., 2005).

Ejemplar examinado: MÉXICO. Guerrero, municipio Zihuatanejo de Azueta, playa El Palmar 02.V.2014, L. Aguilar et al. INV-816 (Invertebrados Asociados a Macroalgas, UNAM).

Rhodophyta

Erythropeltales

Erythrotrichiaceae

*Erythrotrichia tetraseriata N.L. Gardner, Botany 13: 240. 1927. TIPO: ESTADOS UNIDOS DE AMÉRICA. California, San Pedro, H. D. Johnston s.n. (holotipo: UC1110600!).

Filamento unido al sustrato por un disco basal; talo 38-44 $\mu \mathrm{m}$ de diámetro; parte inferior del talo uniseriada, conforme se aproximan al ápice se observan cuatro tiras de células cuadradas a irregulares, 8.5-12.5 $\mu \mathrm{m}$ de diámetro, 10-12.5 $\mu \mathrm{m}$ de largo; cloroplastos en forma de banda.
Distribución: Baja California y Jalisco (Dawson, 1951, 1953a, b, 1961b; Norris, 1975; Abbott y Hollenberg, 1976; Pedroche y González-González, 1981; Pacheco y Aguilar, 1984; Aguilar y Pacheco, 1985; Huerta y Mendoza-González, 1985).

Hábitat: creciendo en sutura de la concha de Chiton articulatus.

Ejemplar examinado: MÉXICO. Guerrero, municipio Zihuatanejo de Azueta, muelle municipal, 01.V.2014, L. Aguilar et al. INV-801 (Invertebrados Asociados a Macroalgas, UNAM).

**Porphyrostromium pulvinatum West \& Zuccarello, Journal of Phycology 47(3): 633. 2011. TIPO: ESTADOS UNIDOS DE AMÉRICA. Monterey County, Carmel, Pebble Beach Bay, N. L. Gardner 2896 (holotipo: UC1110598!). Fig. 2.

Talo pequeño, hasta $1 \mathrm{~mm}$ de largo, con una pequeña extensión rizoidal en la base, hacia la base uniseriado, expansión monostromática local conforme se acerca al ápice; filas 3 células de grosor, células cuadradas 7.5-10 $\mu \mathrm{m}$ de diámetro, 9-12.5 $\mu \mathrm{m}$ de largo.

Distribución: Baja California (Dawson, 1949, 1951, 1953a, b; 1961b; Abbott y Hollenberg, 1976).

Hábitat: creciendo en sutura de Chiton articulatus.

Ejemplar examinado: MÉXICO. Guerrero, municipio Zihuatanejo de Azueta, playa El Palmar, 02.V.2014, L. Aguilar et al. INV-817 (Invertebrados Asociados a Macroalgas, UNAM).

Acrochaetiales

Acrochaetiaceae

***Colaconema coccineum (K.M. Drew) P.W. Gabrielson, Phycological Contribution 6: 95. 2004. TIPO: ESTA- 
DOS UNIDOS DE AMÉRICA. California, San Francisco, Fort Point, 10.IX.1916, N. L. Gardner 3488 (holotipo: UC274015!). Fig. 2.

Talo $0.5 \mathrm{~mm}$ de largo, con ramificación irregular, 1-2 órdenes de ramificación; células 6.25-10 $\mu \mathrm{m}$ de diámetro, 1.5-2.5 diámetros de longitud, disminuye conforme se acerca al ápice; un cloroplasto parietal; monosporangios en las ramillas laterales, pedicelados, 5-7.5 $\mu \mathrm{m}$ de diámetro y 8.5-12.5 $\mu \mathrm{m}$ de largo.

Distribución: costa oeste de Estados Unidos de América y British Columbia, Canadá (Abbott y Hollenberg, 1976).

Hábitat: creciendo en valvas de Chiton articulatus.

Ejemplar examinado: MÉXICO. Guerrero, municipio Zihuatanejo de Azueta, playa El Palmar, 08.I.2014, L. Aguilar et al. INV-796 (Invertebrados Asociados a Macroalgas, UNAM).

\section{Ceramiales}

Rhodomelaceae

*Polysiphonia pacifica Hollenberg. American Journal of Botany 29: 777. 1942. TIPO: ESTADOS UNIDOS DE AMÉRICA. California, Santa Cruz, C. L. Anderson s.n (holotipo: NY!). Fig. 2.

Talo rojo oscuro, unido al sustrato por rizoides, ramificación primaria alterna; ejes 55-180 $\mu \mathrm{m}$ de diámetro, que se atenúan conforme se acerca al ápice; presencia de cuatro células pericentrales, 100-220 $\mu \mathrm{m}$ de diámetro, 4-8 diámetros de longitud; tetrasporangios en series en ramas terminales, 60-70 $\mu \mathrm{m}$ de diámetro.

Distribución: Baja California y Michoacán (Hollenberg, 1942; Dawson 1953b, 1961b; Abbott y Hollenberg, 1976; Huerta, 1978; Aguilar, 1981, 1982; Martinell-Benito, 1983, 1986; Aguilar y Pacheco, 1985; Huerta y Mendoza-González, 1985; Mendoza-González y Mateo-Cid, 1985, 1986; TreviñoMurphy, 1986; Rodríguez, 1989; Sánchez et al., 1989).
Hábitat: creciendo en valvas y suturas de Chiton articulatus.

Ejemplar examinado: MÉXICO. Guerrero, municipio Zihuatanejo de Azueta, playa El Palmar, 20.XI.2014, L. Aguilar et al. INV-852 (Invertebrados Asociados a Macroalgas, UNAM).

\section{Discusión}

La riqueza de especies epizoicas del presente trabajo (124) alcanzó alrededor de $12 \%$ del registro del número de especies del Pacífico mexicano, de 1100 especies (Pedroche y Sentíes, 2003a). Esto sugiere que esta proporción de algas epizoicas podría incrementarse con un mayor énfasis en la recolección, así como con un esfuerzo dirigido a valorar algunos rasgos funcionales, como los hábitos de vida (epizoismo, epifitismo y epilitismo) en las comunidades de macroalgas (Jänes et al., 2017).

De las especies registradas como epizoicas en el Pacífico tropical mexicano, las algas rojas fueron las más diversas, seguidas de las verdes y en menor número las pardas, así como las cianobacterias. Esta relación proporcional entre los diferentes grupos algales coincidió con lo registrado por otros autores para los inventarios florísticos en localidades de distintos estados del Pacífico tropical mexicano (Mateo-Cid y Mendoza-González, 1991, 1992, 2002, 2012; Mendoza-González y Mateo-Cid, 1992, 1996, 1998; Pedroche y Sentíes, 2003a, b; Vega et al., 2008; Dreckmann et al., 2006; Mendoza-González et al., 1994, 2011), e inclusive en las costas del Pacífico donde un estudio a largo plazo demostró que Rhodophyta fue la división más diversa, la cual alcanzó 66.6\% (Lin et al., 2018). Las algas rojas presentan un marcado cambio en su riqueza desde el ecuador hasta mares más fríos, siendo mayor en las zonas tropicales (Lee, 2008), de la misma manera que se presenta en la ficoflora del Pacífico tropical mexicano.

Las familias de algas mejor representadas fueron Corallinaceae, Rhodomelaceae y Cladophoraceae, lo cual coincidió con estudios previos en México (Mateo-Cid y Mendoza-González, 1991, 1992, 2002, 2012; MendozaGonzález y Mateo-Cid, 1992, 1996, 1998; Pedroche y Sentíes, 2003a, b; Mendoza-González et al., 1994, 2011; Vega et al., 2008). 
De las 124 especies de algas reportadas en este estudio, 12 representan nuevos registros para el estado de Guerrero, tres de ellos también lo son para las costas del Pacífico tropical mexicano, así como tres para las costas del Pacífico mexicano. Cabe mencionar que es la primera vez que se registra el género Siphonocladus F. Schmitz para el estado, por lo que se considera importante profundizar en la manifestación de sus caracteres morfológicos, a fin de permitir una adecuada determinación específica, dado que el género ha sufrido importantes cambios nomenclaturales (Guiry y Guiry, 2020).

En cuanto a los nuevos registros para Guerrero, Erythrotrichia tetraseriata estaba registrada para Baja California y Jalisco (Dawson 1951, 1953a, b, 1961b; Norris, 1975; Abbott y Hollenberg, 1976; Pedroche y González-González, 1981; Pacheco y Aguilar, 1984; Aguilar y Pacheco, 1985; Huerta y Mendoza-González, 1985), Polysiphonia pacifica para Baja California y Michoacán (Hollenberg, 1942; Dawson, 1953b, 1961b; Abbott y Hollenberg, 1976; Huerta, 1978; Aguilar, 1981, 1982; Martinell-Benito, 1983, 1986; Aguilar y Pacheco, 1985; Huerta y Mendoza-González, 1985; Mendoza-González y Mateo-Cid, 1985, 1986; Treviño-Murphy, 1986; Rodríguez, 1989; Sánchez et al., 1989), Cladophora laetevirens para el norte del Pacífico tropical mexicano así como en la Península de Baja California (León Tejera et al., 1993; Serviere-Zaragoza et al., 1993, 1998), y Chaetomorpha linum y $C$. californica se registraron previamente en Colima, Michoacán, Nayarit, Oaxaca, Sinaloa y Sonora (MendozaGonzález y Mateo-Cid, 1986; Ortega et al., 1987; Dreckmann et al., 1990; Mateo-Cid y Mendoza-González, 1992; León-Tejera y González-González, 1993; León-Tejera et al., 1993; Serviere-Zaragoza et al., 1993, 1998; Mendoza-González et al., 1994; Dreckmann y Gamboa Contreras, 1998; Aguilar et al., 2002). Finalmente, Ulothrix flacca se había registrado para Michoacán (Stout y Dreckmann, 1993). La ampliación del área de distribución hacia el estado de Guerrero de todas estas especies puede relacionarse con la falta de muestreos en el área de estudio, o a que son individuos de tallas pequeñas como es el caso de las anteriormente mencionadas, lo que confirma la importancia del estudio de otros sustratos como los animales en el conocimiento de la biodiversidad algal.

Como nuevo registro para el Pacífico tropical mexicano se presentan a Cladophora graminea, Urospora wormskioldii y Porphyrostronium pulvinatum, especies previamente registradas para Baja California Norte, Baja California Sur y parte de Sonora (Dawson, 1944, 1945, 1949, 1951, 1953a, b, 1960, 1961b, 1966; Norris, 1975; Abbott y Hollenberg, 1976; Huerta, 1978; Littler y Littler, 1981; Aguilar y Bertsh, 1983; Aguilar y Pacheco, 1986; Aguilar et al., 1990; Pedroche et al., 2005). En relación con los nuevos registros para el Pacífico mexicano, las especies Colaconema coccineum y Urospora penicilliformis han sido reportadas anteriormente solo en localidades templadas del norte y sur del Pacífico americano (Abbott y Hollenberg, 1976). En el caso de Chaetomorpha nodosa, es una especie recientemente señalada como parte de la flora del Atlántico mexicano por QuirozGonzález et al. (2017). La presencia de estas especies en las localidades del estado de Guerrero, además de relacionarse con una falta de muestreo previo en este estado, podría vincularse con la capacidad de dispersión y colonización de estos talos sobre diferentes sitios. Esto ocurre con Urospora penicilliformis, una especie típica de aguas templadas y frías, con un rango de crecimiento óptimo de $5-15{ }^{\circ} \mathrm{C}$, pero que puede tolerar temperaturas de $24-26{ }^{\circ} \mathrm{C}$ (Rico et al., 2003). Su llegada a las costas del Pacífico tropical mexicano podría explicarse por su capacidad para tolerar diversos rangos de radiación UV y de temperatura superiores al óptimo (Roleda et al., 2009). En Guerrero, estado donde se hace el registro de esta especie, los rangos de temperatura del agua oscilan entre $25-28{ }^{\circ} \mathrm{C}$ en la temporada de secas, pudiendo alcanzar hasta $30^{\circ} \mathrm{C}$ en lluvias, momento del año en el que se registró dicha especie, lo que indicó que ésta ha alcanzado rangos de tolerancia superiores a los registrados previamente por Rico et al. (2003). Además, cabe mencionar que no es la primera vez que se registra en aguas tropicales de México, ya que García-López et al. (2017) la ubicaron en las costas de Veracruz. Esto también ocurre con Chaetomorpha nodosa, especie previamente registrada en el Golfo de México por Quiroz et al. (2017). La ampliación de la distribución de especies previamente registradas en ambientes templados podría vincularse al transporte accidental de organismos como parte de la flora y fauna incrustantes en los cascos de embarcaciones que se trasladan constantemente entre continentes y regiones (Aguilar et al., 2014).

Es importante destacar que Chaetomorpha linum es la especie más comúnmente registrada como epizoica, 
tanto en vertebrados como en invertebrados en distintos trabajos (Sentíes et al., 1999; Báez, 2002; Levenets, 2010; Martins et al., 2014), en regiones templadas y tropicales, cuya presencia puede deberse a su condición como especie oportunista (Sorce et al., 2018), misma que además puede ser indicadora de ambientes eutrofizados. En este trabajo, C. linum se registró en el muelle municipal que, de acuerdo con otros autores (Aguilar-Estrada et al., 2017; López et al., 2017), es un área contaminada y con altas tasas de sedimentación, por lo que la presencia de esta especie de alga verde podría ser un indicador de procesos de eutrofización.

Las diferencias entre las especies de algas registradas en Chiton articulatus y las reportadas en la literatura como epizoicas se deben a que estas últimas fueron registradas en mayor número de sustratos y localidades, sin restringirse a una sola especie de basibionte como es la cucaracha de mar.

Principalmente las especies Cladophora laetevirens, Chaetomorpha linum, Urospora penicilliformis y Ulothrix flacca, mencionadas como nuevos registros, han sido objeto de controversias taxonómicas, por lo que es importante considerar futuros tratamientos moleculares y morfológicos para tener certidumbre de la identidad de esos taxones.

Guerrero presentó el mayor número de registros de algas epizoicas, lo que representa $36 \%$ del inventario florístico para el estado de acuerdo con otros autores (Pedroche y Sentíes, 2003a; Mateo-Cid y Mendoza-González, 2012). A pesar de que Pedroche y Sentíes (2003b) mencionaron que los estados con la mayor riqueza de algas son Oaxaca (222) y Nayarit (213), estos no presentaron tantos registros de algas epizoicas. Esto fue probablemente causado por los diferentes objetivos de los estudios y en consecuencia por los diferentes tipos de muestreos seguidos, enfocados principalmente a la recolección de algas en el intermareal rocoso para la elaboración de ficofloras que dejan de lado a otros sustratos como los animales.

De las especies registradas en este trabajo, algunas han sido señaladas como epizoicas en otras regiones del mundo, donde la interacción se ha documentado mayormente entre las algas y organismos vertebrados como tortugas y peces (Ballantine et al., 2001; Báez et al. 2002; Báez y Flores-Moya, 2003).

Por ejemplo, se comparten seis especies del presente trabajo con el estudio llevado a cabo por Ballantine et al.
(2001) de algas epizoicas en peces escorpión en Puerto Rico (Scytonematopsis crustacea (Thuret ex Bornet \& Flahault) Koválik \& Komárek, Centroceras clavulatum, Champia parvula, Chroodactylon ornatum (C. Agardh) Bason, Erythrotrichia carnea (Dillwyn) C. Agardh y Sahlingia subintegra (Rosenvinge) Kornmann), mientras que al comparar con los trabajos realizados por Báez et al. (2002) y Báez y FloresMoya (2003) de la flora que crece en peces espada y tortugas del Mediterráneo, sólo hubo tres especies coincidentes (Chaetomorpha linum, Ectocarpus siliculosus y Sphacelaria tribuloides).

Además, se han llevado a cabo varios trabajos que han evaluado la interacción entre algas y moluscos, sustrato que de acuerdo con Wahl (2008) es uno de los más recurrentes para las algas. Levenets et al. (2010) observaron sobre moluscos pectínidos de Japón, 51 especies de algas epibiontes, de las cuales Chaetomorpha linum, Derbesia marina (Lyngbye) Soliery y Erythrotrichia carnea coinciden con el presente estudio. Así mismo, sobre Ceratozona squalida C.B. Adams 1845 de Florida (Connelly y Turner, 2009), se registraron 27 especies de algas epizoicas de las cuales Chaetomorpha antennina y Ulva flexuosa Wulfen también se presentan en este trabajo. Chaetomorpha linum y Cladophora laetevirens, especies consideradas cosmopolitas, fueron igualmente registradas en Argentina como epibiontes de la lapa Patella aspera Röding 1798 por Martins et al. (2014).

Respecto a los tres estudios realizados en México, Sentíes et al. (1999) reportaron a Chaetomorpha linum, Sphacelaria tribuloides, Centroceras clavulatum, Erytrotrichia carnea y Champia parvula sobre tortugas marinas en el Caribe. Estas algas también fueron registradas en el presente estudio. Hernández-Vázquez y Valadez-González (1998), y recientemente Álvarez-Cerillo et al. (2017), registraron a las algas a nivel de grupo morfofuncional sin ahondar en la identidad de los taxones, destacando la presencia de filamentos y costras en tortugas marinas y quitones, respectivamente. Por otro lado, es importante mencionar que en algunos estudios no se especifica el tipo de sustrato sobre el que se encuentran las algas y es aún más frecuente que se proporcionen datos acerca de especies que son epífitas que aquellas que son epizoicas.

En este estudio solo 17 especies epizoicas aparecieron sin descripción del basibionte y fueron los moluscos los 
que presentaron la mayor cantidad de especies algales epizoicas, sin especificación de los taxa incluidos, a excepción de Chiton articulatus estudiado aquí.

Con relación a los grupos morfofuncionales que presentaron las algas epizoicas aquí registradas (124 especies), la gran mayoría se encuentra entre los filamentos (43.6\%), los filamentos corticados $(20.6 \%)$ y las costras $(15.8 \%)$, sumando $80 \%$ del total. La presencia mayoritaria de los filamentos indica ensambles algales soportados por especies anuales y oportunistas de rápido crecimiento, complementados por especies perennes, sucesionales tardías y de crecimiento lento como son los filamentos corticados y las costras (Steneck y Dethier, 1994).

Debido a que la mayor parte de las algas bentónicas se adhieren al sustrato por medio de distintas estructuras como rizoides, discos y hápteros, o a partir de sustancias cementantes (algas pardas costrosas), su presencia en la mayoría de los casos está asociada a distintas rocas y guijarros, donde diferentes características, como la estructura física del sustrato, la dureza y el grado de compactación, juegan un papel muy importante en la distribución de las algas marinas (Santelices, 1977). Esto puede explicar la predominancia de los moluscos como sustratos para las algas, ya que las conchas de estos organismos presentan un alto grado de dureza debido a su conformación con carbonato de calcio y proteínas (Hickman, 2013; Heller, 2015).

Por otra parte, se han reportado especies de algas creciendo sobre coral vivo (Vázquez-Texocotitla, 2013); estos parecen representar un sustrato óptimo para el establecimiento de algas (Díaz-Pulido y McCook, 2004). Se registraron seis especies de algas creciendo sobre esponjas, las cuales tienen una superficie bastante heterogénea en términos de relieve, por lo que puede proporcionar a las algas un sitio adecuado para el desarrollo de los propágulos y germinación de las esporas, así como un espacio para la protección y fijación de los talos, reduciendo el desprendimiento de estos por el efecto de las olas y las corrientes (Arias et al., 2006; Passarelli et al., 2014; Gastaldi et al., 2015).

Generalmente, los epibiontes presentan tallas pequeñas lo que los convierte en organismos poco conspicuos al momento de realizar un inventario florístico (Mendoza-González et al., 2011), por lo que muy probable- mente su abundancia y diversidad han sido subestimadaa. Esto coincide con que gran parte de las especies registradas como epizoicas en el presente trabajo son pequeñas, incluyendo entre ellas a la mayoría de las especies de la familia Rhodomelaceae, que es la mejor representada en cuanto a número de especies.

\section{Conclusiones}

La epibiosis constituye un componente importante en el estudio de la estructura de las comunidades marinas bentónicas; sin embargo, son escasos los estudios realizados, siendo predominantes los reportes de epifitismo y en muy pocos casos de los organismos epizoicos. Por ello es importante desarrollar más estudios que se enfoquen al conocimiento de este tipo de epibiosis algal.

Los resultados de este trabajo muestran que la biodiversidad de algas presente en los sustratos animales es alta; además contribuye notablemente a la riqueza específica registrada para Guerrero y para el Pacífico de México. Se hace patente la importancia de desarrollar más estudios que se enfoquen al conocimiento de la epibiosis algal, además de la perspectiva florística, desde el punto de vista de las interacciones ecológicas.

\section{Contribución de autores}

LA, IR y DR llevaron a cabo las recolecciones del material en las áreas de muestreo. NQ realizó la revisión del material muestreado, la elaboración de preparaciones, la toma de medidas, y la identificación y descripción de los ejemplares. NQ y LA llevaron a cabo la revisión bibliográfica. NQ y LA escribieron el manuscrito con el apoyo de DR e IR. Todos los autores contribuyeron a la discusión, revisión y aprobación del manuscrito final.

\section{Agradecimientos}

Agradecemos las facilidades y apoyo otorgados en la Unidad Multidisciplinaria de Investigación y Docencia Zihuatanejo (UMDI-Z), Universidad Nacional Autónoma de México (UNAM), a cargo de Norma López, y en particular a Carlos Candelaria por su apoyo técnico en campo. Agradecemos a Ana I. Bieler (Facultad de Ciencias-UNAM) por las fotografías en este trabajo. 


\section{Financiamiento}

Este trabajo fue financiado por un proyecto DGAPA-PAPIIT, UNAM (IN220714).

\section{Literatura citada}

Abbott, I. A. y G. J. Hollenberg. 1976. Marine algae of California. Stanford University Press. Stanford, USA. 789 pp.

Aguilar, L. 1981. Algas rojas (Rhodophyta) de la Bahía Todos Santos, Baja California, México, durante el ciclo anual 19781979. Ciencias Marinas 7(1): 83-101. DOI: https://doi. org/10.7773/cm.v7i1.356

Aguilar, L. 1982. Ocurrencia de las algas cafés (Phaeophyta) en la Bahía Todos Santos, Baja California. Ciencias Marinas 8: 25-34. DOI: https://doi.org/10.7773/cm.v8i2.401

Aguilar, L. y H. Bertsh. 1983. Algas verdes (Chlorophyta) de la Bahía de Todos Santos, Baja California, México. Ciencias Marinas 9: 111-124. DOI: https://doi.org/10.7773/cm.v9i1.408

Aguilar, L. e I. Pacheco. 1985. Nuevos registros y ampliación de rango geográfico para algas marinas de la costa del Pacífico de Baja California, México. II. Ciencias Marinas 11(2): 6976. DOI: https://doi.org/10.7773/cm.v11i2.466

Aguilar, L., F. Pedroche y J. Zertuche-González. 2014. Macroalgas marinas introducidas en la costa del Pacífico de México. Estado actual. In: Low-Pfeng, A., P. Quijón y E. Peters-Recagno (eds.). Especies invasoras acuáticas: casos de estudio en ecosistemas de México. Secretaria de Medio Ambiente y Recursos Naturales, Instituto Nacional de Ecología y Cambio Climático (INECC) University of Prince Edward Island (UPEI, Canada). México, D.F., México. Pp. 95-117.

Aguilar, L. E., R. Aguilar, L. Mateo-Cid y A. C. Mendoza González. 2002. Marine algae from the Gulf of Santa Clara, Sonora, México. Hydrobiologia 477: 231-238.

Aguilar, R. e I. Pacheco. 1986. Variaciones estacionales de las algas verdes (Chlorophyta) de la costa Noroccidental de la Península de Baja California. Ciencias Marinas 12(1): 73-78. DOI: https://doi.org/10.7773/cm.v12i1.484

Aguilar, R., I. Pacheco y L. Aguilar. 1990. Algas marinas de las Islas Todos Santos, Baja California, México. Ciencias Marinas 16(2): 117-119. DOI: https://doi.org/10.7773/cm.v16i2.687

Aguilar-Estrada, L., I. Ruiz-Boijseauneau y D. Rodríguez. 2017. Estadios juveniles de las especies de gasterópodos pateliformes y de poliplacóforos (Mollusca) asociados a macroalgas intermareales de Guerrero, México. Revista Mexi- cana de Biodiversidad 88(2): 280-299. DOI: https://doi. org/10.1016/j.rmb.2017.03.021

Álvarez-Cerrillo, L., P. Valentich-Scott y W. Newman. 2017. A remarkable infestation of epibionts and endobionts of an edible chiton (Polyplacophora: Chitonidae) from the Mexican tropical Pacific. The Nautilus 131(1): 87-96.

Anagnostidis, K. y J. Komárek. 1988. Modern approach to the classification system of Cyanophytes. 3. Oscillatoriales. Archv fur Hydrobiologie 80(1-4): 327-472.

Arias, J., S. Zea, F. Newmark y M. Santos-Acevedo. 2006. Determinación de la capacidad antiepibiótica de los extractos orgánicos crudos de las esponjas marinas Cribrochalina infundibulum y Biemna cribaria. Boletín de Investigaciones Marinas y Costeras 35: 91-101.

Ávila-Torres, E. 2006. Ecología de la asociación Haliclona caerulea (Porifera: Demospongiae) y Jania adherens (Rhodophyta: Corallinales) en la Bahía de Mazatlán. Tesis de doctorado. Posgrado en Ciencias del Mar y Limnología. Mazatlán, México. 243 pp.

Báez, J. C. y A. Flores-Moya. 2003. Macroalgas epibiónticas. https://www.researchgate.net/publication/317640939_ Macroalgas_epibionticas (consultado agosto de 2018).

Báez, J., J. Camiñas, J. Valeiras, F. Conde y A. Flores-Moya. 2001. First record of the epizoic red seaweed Polysiphonia carettia Hollenberg in the Mediterranean Sea. Acta Botanica Malacitana 26: 197-201. DOI: https://doi.org/10.24310/ abm.v26i0.7417

Báez, J., J. Camiñas, J. Valeiras, F. Conde y A. Flores-Moya. 2002. Preliminary check-list of the epizootic macroalgae growing on loggerhead turtles in the Western Mediterranean Sea. Marine Turtle Newsletter 98: 1-2.

Ballantine, D., N. Navarro y D. Hensley. 2001. Algal colonization of Caribbean scorpion fishes. Bulletin of Marine Science 69(3): 1089-1094.

Bretos, M. y R. Chihuailaf. 1990. Biometría y otros aspectos biológicos de Fissurella pulchra (Mollusca: Prosobranchia). Biología Marina 25(1): 1-14.

Connelly, P. W. y R. L. Turner. 2009. Epibionts of the Eastern Surf Chiton, Ceratozona squalida (Polyplacophora: Mopaliidae), from the Atlantic Coast of Florida. Bulletin of Marine Science 85(3): 187-202.

Dawson, E. Y. 1944. The marine algae of the Gulf of California. Allan Hancock Pacific Expedition 3(10): 189-464. 
Dawson, E. Y. 1945. Notes on Pacific coast marine algae II. Bulletin of the Southern California Academy of Sciences 44(1): 22-27.

Dawson, E. Y. 1949. Resultados preliminares de un reconocimiento de las algas marinas de las costas Pacífica de México. Revista de la Sociedad Mexicana de Historia Natural 9: 215-255.

Dawson, E. Y. 1951. A further study of upwelling and associated vegetation along Pacific Baja California, Mexico. Journal of Marine Research 10(1): 39-58.

Dawson, E. Y. 1953a. Marine red algae of Pacific Mexico. I Bangiales to Corallinoideae. Allan Hancock Pacific Expeditions 17(1): 1-239.

Dawson, E. Y. 1953b. Resumen de las investigaciones recientes sobre algas marinas de la costa pacífica de México, con una sinopsis de la literatura, sinonimia y distribución de las especies descritas. Revista de la Sociedad Mexicana de Historia Natural 13: 97-197.

Dawson, E. Y. 1954. Marine red algae of Pacific Mexico. II Cryptonemiales. Allan Hancook Pacific Expeditions 17(2): 241-397.

Dawson, E. Y. 1960. Marine red algae of Pacific Mexico. III Cryptonemiales. Corallinaceae, subfamily Melobesioideae. Pacific Naturalist 2(1): 1-125.

Dawson, E. Y. 1961a. Marine red algae of Pacific Mexico. IV Gigartinales. Pacific Naturalist 2(5): 191-341.

Dawson, E. Y. 1961b. A guide to the literature and distributions of Pacific benthic algae from Alaska to the Galapagos Islands. Pacific Science 15: 370-461.

Dawson, E. Y. 1962. Una clave ilustrada de los géneros de algas bénticas del Pacífico de la América Central. Pacific Naturalist 3: 167-231.

Dawson, E. Y. 1963a. Marine red algae of Pacific Mexico. VI Rhodymeniales. Nova Hedwigia 5: 437-476.

Dawson, E. Y. 1963b. Marine red algae of Pacific Mexico. VIII Ceramiales, Dasyaceae, Rhodomelaceae. Nova Hedwigia 6: 401-481.

Dawson, E. Y. 1966. New records of marine algae from the Gulf of California. Journal of the Arizona Academic of Science 4(2): 55-66.

Díaz-Pulido, G. y L. J. McCook. 2004. Effects of live coral, epilithic algal communities and substrate type on algal recruitment. Coral Reefs 23(2): 225-233. DOI: https://doi.org/10.1007/ s00338-004-0370-5

Dreckmann, K. 1987. Algas marinas bénticas de Playa San Telmo, Michoacán, México. Tesis profesional. Facultad de Ciencias,
Universidad Nacional Autónoma de México. México, D.F., México. 170 pp.

Dreckmann, K. M. y J. A. Gamboa Contreras. 1998. Ficoflora marina bentónica actualizada del Golfo de Tehuantepec y algunos registros para Guatemala. In: Tapia García, M. (ed.). El Golfo de Tehuantepec: El ecosistema y sus recursos. Universidad Autónoma Metropolitana-Iztapalapa. México, D.F., México. 75-91 pp.

Dreckmann, K. M., F. Pedroche y A. Sentíes. 1990. Lista florística de las algas marinas bentónicas de la costa norte de Michoacán, México. Boletín de la Sociedad Botánica de México. 50: 19-42. DOI: https://doi.org/10.17129/botsci.1375

Dreckmann, K. M., A. Sentíes G., F. F. Pedroche y M. Callejas. 2006. Diagnostico florístico de la ficología marina bentónica en Chiapas. Hidrobiológica 16(2):147-158.

Frazier, J., D. Margaritoulis, K. Muldoon, C., Potter, J. Rosewater, C. Ruckdeschel y S. Salas. 1985. Epizoan communities on marine turtles: I Bivalve and Gastropod mollusks. Marine Ecology 6(2): 127-140. DOI: https://doi. org/10.1111/j.1439-0485.1985.tb00134.x

García-López, D., L. Mateo-Cid y C. Mendoza-González. 2017. Nuevos registros y lista actualizada de las algas verdes (Chlorophyta) del litoral de Veracruz, México. Gayana Botánica 74(1): 41-56. DOI: https://doi.org/10.4067/s071766432017005000104

Gastaldi, M., F. N. Firstater, P. Daleo y M. A. Narvarte. 2015. Abundance of the sponge Hymeniacidon cf. perlevis in a stressful environment of Patagonia: relationships with Ulva lactu$c a$ and physical variables. Journal of the Marine Biological Association of the United Kingdom 96(2): 465-472. DOI: https://doi.org/10.1017/S0025315415001198

González-González, J., M. Gold-Morgan, H. León-Tejeda, C. Candelaria, D. León-Álvarez, E. S. Zaragoza y D. Fragoso. 1996. Catálogo onomástico (nomenclator) y bibliografía indexada de las algas bentónicas marinas de México. Cuadernos No. 34. Instituto de Biología, Universidad Nacional Autónoma de México. México, D.F., México. 491 pp.

Guiry, M. D y W. D. Guiry. 2020. AlgaeBase version 4.2. World-wide electronic publication, National University of Ireland, Galway. http://www.algaebase.org (consultado marzo de 2020).

Heller, J. 2015. What is a snail? In: Heller, J. (ed.). Sea snails a natural history. Springer. Nueva York, USA. Pp. 12-17. DOI: https://doi.org/10.1007/978-3-319-15452-7_2 
Hernández-Vázquez, S. y C. Valadez-González. 1998. Observaciones de los epizoarios encontrados sobre la tortuga golfina Lepidochelys olivacea en La Gloria, Jalisco, México. Ciencias Marinas 24(1): 119-125.

Hickman, C. S. 2013. Interacting constraints and the problem of similarity in gastropod structure and function. American Malacological Bulletin 31(1): 155-168.

Hollenberg, J. 1942. Phycological notes-I. Bulletin of the Torrey Botanical Club 68(7): 528-538.

Huerta, L. 1978. Vegetación marina litoral. In: Rzedowski, J. (ed.). Vegetación de México. Editorial Limusa. México, D.F., México. Pp. 328-340.

Huerta, L. y Mendoza-González. 1985. Algas marinas de la parte sur de la Bahía de la Paz, Baja California Sur. Phytologia 59(1): 35-57.

Jänes, H., J. Kotta, M. Pärnoja, T. P. Crowe, F. Rindi y H. Orav-Kotta. 2017. Functional traits of marine macrophytes predict primary production. Functional Ecology 31(4): 975-986. DOI: https://doi.org/10.1111/1365-2435.12798

Kaas, P., R. Van Belle y H. Strack. 2006. Monograph of Living Chitons (Mollusca: Polyplacophora). Suborder Ischnochitonina (concluded): Schizochitonidae and Chitonidae. Additions to Volumes 1-6. Brill Academic Publishers. Leiden, Netherlands.

Keen A. M. 1971. Sea Shells of Tropical West America Marine mollusks from Baja California to Peru. California: Stanford University Press. Stanford, USA.

Lee, R. 2008. Phycology. Cambridge. New York, USA. 561 pp.

León-Tejera, H. y J. González-González. 1993. Macroalgas de Oaxaca. In: Salazar-Vallejo, S. I. y N. E. González (eds.). Biodiversidad marina y costera de México. Comisión Nacional para el Conocimiento y Aprovechamiento de la Biodiversidad y Centro de Investigaciones de Quintana Roo (CIQRO). Chetumal, México. 865 pp.

León-Tejera, H., D. Fragoso, D. Álvarez, C. Candelaria, E. Serviere y J. González-González. 1993. Characterization of tidal pool algae in the Mexican Tropical Pacific coast. Hydrobiologia 260: 197205. DOI: https://doi.org/10.1007/BF00049020

León-Tejera, H., E. Serviere-Zaragoza y J. González-González. 1996. Affinities of the marine flora of the Revillagigedo islands, México. Hydrobiologia 326: 159-168. DOI: https://doi. org/10.1007/BF00047801

Levenets, I. R., I. I. Ovsyannikova y E. B. Lebedev. 2010. Epibiotic Macroalgae on the scallop Mizuhopecten yessoensis in
Peter the Great Bay, Sea of Japan. Russian Journal of Marine Biology 36(5): 340-349. DOI: https://doi.org/10.1134/ s1063074010050032

Lin, S. M., L. C. Tseng, P. O. Ang, J. Bolton y L. Liu. 2018. Long-term study on seasonal changes in floristic composition and structure of marine macroalgal communities along the coast of Northern Taiwan, southern East China Sea. Marine Biology 165: 1-17. DOI: https://doi.org/10.1007/s00227-018-3344-9 Littler, M. y D. Littler. 1981. Intertidal Macrophyte Communities from Pacific Baja California and the Upper Gulf of California: Relatively Constant vs. Environmentally Fluctuating Systems. Marine Ecology Progress Series 4: 145-158. DOI: https://doi. org/10.3354/meps004145

López, N., C. Candelaria, P. Ramírez-García y D. Rodríguez. 2017. The structure of tropical turf-forming algae assemblages. Zihuatanejo Bay, México. Latin American Journal of Aquatic Research 45(2): 329-340. DOI: https://doi.org/10.3856/ vol45-issue2-fulltext-9

Luna-Barreda, L. J. 2018. Variación de las relaciones espaciales alga-coral durante el fenómeno de El Niño (2015-2016) en Ixtapa - Zihuatanejo, Guerrero, México. Tesis de licenciatura. Facultad de Ciencias, Universidad Nacional Autónoma de México. Cd. Mx., México. 91 pp.

Martinell-Benito, L. 1983. Estudio prospectivo de las algas rojas (Rhodophyta) de las desembocaduras del rio Balsas. Tesis de licenciatura. Facultad de Ciencias, Universidad Nacional Autónoma de México. Cd. Mx., México. 97 pp.

Martinell-Benito, L. 1986. Estudio ecológico de las algas de las desembocaduras de Michoacán. Tesis de maestría. Facultad de Ciencias, Universidad Nacional Autónoma de México. Cd. Mx., México. 179 pp.

Martins, G. M., J. Faria, M. Furtado y A. I. Neto. 2014. Shells of Patella aspera as 'islands' for epibionts. Journal of the Marine Biology Association of the United Kingdom 94: 1027-1032. DOI: https://doi.org/10.1017/S002531541400044

Mateo-Cid, L. E. y A. C. Mendoza-González. 1991. Algas marinas bénticas de la costa del estado de Colima. Acta Botanica Mexicana 13: 9-30. DOI: https://doi.org/10.21829/ abm13.1991.605

Mateo-Cid, L. E. y A. C. Mendoza-González. 1992. Algas marinas bentónicas de la costa sur de Nayarit, México. Acta Botanica Mexicana 20: 13-28. DOI: https://doi.org/10.21829/ abm20.1992.653 
Mateo-Cid, L. E. y A. C. Mendoza-González. 1997. Nuevos registros de algas marinas para Oaxaca, México. Polibotánica 4: 54-74.

Mateo-Cid, L. E. y A. C. Mendoza-González. 2002. Algas marinas bentónicas de la costa de Oaxaca, México. Anales de la Escuela Nacional de Ciencias Biológicas 47: 11- 23.

Mateo-Cid, L. E. y A. C. Mendoza-González. 2012. Algas marinas bentónicas de la costa noroccidental de Guerrero, México. Revista Mexicana de Biodiversidad 83(4): 905-928. DOI: https://doi.org/10.22201/ib.20078706e.2012.4.1010

Mendoza-González, A. C. y L. E. Mateo-Cid. 1985. Contribución al estudio florístico de la costa occidental de Baja California, México. Phytologia 59: 17-33.

Mendoza-González, A. C. y L. E. Mateo-Cid. 1986. Flora marina bentónica de la costa noroeste del estado de Sonora, México. Phytologia 60: 414-427.

Mendoza-González, A. C. y L. E. Mateo-Cid. 1992. Estudio preliminar de las algas marinas bentónicas de la costa de Jalisco, México. Anales de la Escuela Nacional de Ciencias Biológicas 37: $9-25$

Mendoza-González, A. C. y L. E. Mateo-Cid. 1996. Contribución al estudio de la ficoflora marina de la costa del estado de Chiapas, México (parte a). Polibotánica 2: 61-118.

Mendoza-González, A. C. y L. E. Mateo-Cid. 1998. Avance de un estudio sobre las macroalgas marinas de Guerrero y Oaxaca. Ciencia y Mar 4: 15-29.

Mendoza-González, A. C., L. E. Mateo-Cid y L. Huerta. 1994. Algas marinas de Mazatlán, Sinaloa, México. Acta Botanica Mexicana 27: 99-115. DOI: https://doi.org/10.21829/ abm27.1994.713

Mendoza-González, A. C., L. E. Mateo-Cid y C. Galicia-García. 2011. Integración florística de las algas marinas de la costa sur de Jalisco, México. Revista Mexicana de Biodiversidad 82(1): 1949. DOI: https://doi.org/10.22201/ib.20078706e.2011.1.383

Moncada-García, A. I. 2018. Evaluación del impacto de la sedimentación en la estructura de los ensambles algales del arrecife en Caleta de Chon de Zihuatanejo, Guerrero, México. Tesis de licenciatura. Facultad de Ciencias, Universidad Nacional Autónoma de México. Cd. Mx., México. 75 pp.

Norris, J. N. 1975. Marine Algae of the Northern Gulf of California. PhD. Disertation. University of California. Santa Barbara, USA. $575 \mathrm{pp}$.

Ortega, M., J. Ruíz Cárdenas y G. Oliva Martínez. 1987. La vegetación sumergida en la Laguna Agiabampo, Sonora-Sinaloa.
Anales del Instituto de Biología. Universidad Nacional Autónoma de México, Serie Botánica 57: 59-108.

Pacheco, I. y L. Aguilar. 1984. Distribución estacional de Rhodophyta en el noroeste de Baja California. Ciencias Marinas 10: 67-80.

Passarelli, C., F. Olivier, D. M. Paterson, T. Meziane y C. Hubas. 2014. Organisms as cooperative ecosystem engineers in intertidal flats. Journal of Sea Research 92: 92-101. DOI: https://doi.org/10.1016/j.seares.2013.07.010

Pedroche, F. y J. González-González. 1981. Lista preliminar de las algas marinas de la región sur de la costa de Jalisco, México. Phycological Latin America 1: 60-72.

Pedroche, F. y A. Sentíes. 2003a. El litoral del Pacífico mexicano y su exploración ficológica. Contribuciones ficológicas de México. Sociedad Ficológica de México, AC. Mérida, México. Pp. 5-11.

Pedroche, F. y A. Sentíes. 2003b. Ficología marina mexicana. Diversidad y Problemática actual. Hidrobiológica 13(1): 23-32.

Pedroche, F., P. C. Silva, L. E. Aguilar-Rosas, K. M. Dreckmann y R. Aguilar-Rosas. 2005. Catálogo de las algas marinas bentónicas del Pacífico de México. I. Chlorophycota. Universidad Autónoma Metropolitana, Universidad Autónoma de Baja California, University of California. México, D.F., México. $135 \mathrm{pp}$.

Pérez-García, M. 1967. Algas de la familia Corallinaceae (División Rhodophyta) de la Bahía de Zihuatanejo. Tesis de licenciatura. Facultad de Ciencias, Universidad Autónoma de México. México, D.F., México. 115 pp.

Ponce-Márquez, E. 2010. Gelidium (Gelidiales, Rhodophyta) del Pacífico tropical mexicano: variación genética inter e intraespecífica. Tesis de doctorado. Universidad Nacional Autónoma de México. Cd. Mx., México. 142 pp.

Quiroz-González, N., D. León-Álvarez y M. G. Rivas-Acuña. 2017. Nuevos registros de algas verdes marinas (Ulvophyceae) para Tabasco, México. Acta Botanica Mexicana 118: 121138. DOI: https://doi.org/10.21829/abm118.2017.1204

Rico, A., P. Lanas y J. López-Gappa. 2003. Colonization of Ulothrix flacca, Urospora penicilliformis and Blidigia marginata (Chlorophyta) in Comodoro Rivadavia harbor (Chubut, Argentina). Revista del Museo Argentino de Ciencias Naturales nueva serie 5: 93-97.

Rodríguez, D. 1989. Gelidiales-Rhodophyta: una contribución a la flora tónica del Pacífico tropical mexicano. Propuesta teórico-metodológica a partir de la teoría de procesos alterados. 
Tesis de doctorado. Facultad de Ciencias, Universidad Nacional Autónoma de México. México, D.F., México. 397 pp.

Rodríguez, D., N. López y J. González-González. 2008. Gelidiales (Rhodophyta) en las costas del Pacífico mexicano con énfasis en las especies tropicales. In: Sentíes, A. y K. Dreckmann (eds.). Monografías Ficológicas Vol. 3. Universidad Autónoma Metropolitana. México, D.F., México. Pp. 27-74.

Roleda, M., G. Campana, C. Wiencke, D. Hanelt, M. Quartino y A. Wulff. 2009. Sensitivity of Antarctic Urospora penicilliformis (Ulotrichales, Chlorophyta) to ultraviolet radiation is lifestage dependent. Journal of Phycology 45(3): 600-609. DOI: https://doi.org/10.1111/j.1529-8817.2009.00691.x

Santelices, B. 1977. Ecología de algas marinas bentónicas. Documento de la Dirección General de Investigaciones. Universidad Católica de Chile. Santiago de Chile, Chile. 488 pp.

Sánchez, I., C. Fajardo y C. Oliveiro. 1989. Estudio florístico estacional de las algas en Bahía Magdalena, B.C.S., México. Investigaciones marinas CICIMAR 4: 35-48.

Sandoval-Coronado, B. A. 2016. Patrones de abundancia y distribución de macroalgas del arrecife coralino de Isla Ixtapa, Zihuatanejo, Guerrero. Tesis de licenciatura. Facultad de Ciencias, Universidad Nacional Autónoma de México. Cd Mx., México. 75 pp.

Sentíes, A., J. Espinoza-Ávalos y J. Zurita. 1999. Epizoic algae of nesting sea turtles Caretta caretta and Chelonia mydas from the Mexican Caribbean. Bulletin of Marine Science 64(1): 185-189.

Serio, D., M. Catra, D. Collodoro y A. Nisi. 2011. Ceramium cormacii sp. nov. (Ceramiaceae, Rhodophyta), a new Mediterranean species epizoic on logger head sea turtles (Caretta caretta). Botanica Marina 54(6): 545-550. DOI: https://doi. org/10.1515/bot.2011.068

Serviere-Zaragoza, E., J. González-González y D. Rodríguez. 1993. Ficoflora de la región de Bahía Banderas, Jalisco-Nayarit. In: Salazar-Vallejo, S. I. y N. E. González (eds.). Biodiversidad marina y costera de México. Comisión Nacional para el Conocimiento y Uso de la Biodiversidad y Centro de Investigaciones de Quintana Roo. Chetumal, México. Pp. 475-485.

Serviere-Zaragoza, E., S. Castillo Arguero y J. González-González. 1998. Descripción ficológica de los ambientes de la región de bahía de Banderas, Nayarit-Jalisco, México. Boletín del Instituto de Botánica (Universidad de Guadalajara) 5: 157 180.
Steneck, R. S. y M. N. Dethier. 1994. A Functional Group Approach to the Structure of Algal-Dominated Communities. Oikos 69(3): 476-498. DOI: https://doi.org/10.2307/3545860

Sorce, C., M. Persiano y M. Lenzi. 2018. Growth and physiological features of Chaetomorpha linum (Müller) Kütz. in high density mats. Marine Pollution Bulletin 129(2): 772-781. DOI: https://doi.org/10.1016/j.marpolbul.2017.10.071

Stout, I. y K. M. Dreckmann. 1993. Macroalgas bentónicas de faro de Bucerías, Michoacán, México. Anales del Instituto de Biología, Serie Botánica 64: 1-23.

Taylor, W. R. 1945. Pacific marine algae of the Allan Hancock Expeditions to the Galapagos Islands. Allan Hancock Pacific Expeditions 12: 1-528.

Taylor, W. R. 1960. Marine algae of the eastern tropical and subtropical coast of the Americas. University of Michigan Press. Ann Arbor, USA. 870 pp.

Treviño-Murphy, L. 1986. Estudio ficoflorístico del ambiente marino escollera en la zona costera de Lázaro Cárdenas, Michoacán. Tesis de licenciatura. Facultad de Ciencias. Universidad Nacional Autónoma de México. México, D.F., México. 92 pp.

Vázquez-Delfín, E. 2008. Diagnóstico general de la biodiversidad marina de la región de Ixtapa-Zihuatanejo, Guerrero: bases para su conservación. Tesis de licenciatura. Facultad de Ciencias, Universidad Nacional Autónoma de México. Cd. Mx., México. 193 pp.

Vázquez-Texocotitla, P. 2013. Variación espacio-temporal de la estructura de los ensambles asociados a diferentes sustratos en el arrecife coralino El Zacatoso, Zihuatanejo, Guerrero. Tesis de maestría, Universidad Nacional Autónoma de México. Cd. Mx., México. 121 pp.

Vega, C., C. Olabarría y J. L. Carballo. 2008. Variación espacio-temporal de moluscos y macroalgas en sustratos rocosos intermareales en la bahía de Mazatlán. Ciencia y Mar 34: 3-16.

Wahl, M. 2008. Ecological lever and interface ecology: epibiosis modulates the interactions between host and environment. Biofouling 24(6): 427-438. DOI: http://doi. org/10.1080/08927010802339772

Wahl, M. 2009. Epibiosis: Ecology, Effects and Defence. In: Dürr, S. y J. C. Thomason (eds.). Marine Hard Bottom Communities. Springer-Verlag. Heidelberg, Germany. Pp. 61-72. DOI: https://doi.org/10.1002/9781444315462.ch7 
Apéndice: Especies de algas epibiontes registradas sobre la concha del molusco Chiton articulatus G.B. Sowerby, de acuerdo con los muestreos de este trabajo y con la literatura consultada. Estado: I. Sin.; II. Nay.; III. Jal.; IV. Col.; V. Mich.; VI. Gro.; VII. Oax.; VIII. Chis. Grupo morfofuncional (GMF): C. Costra; CA. Coralina articulada; F. Filamento; FC. Filamento corticado; FO. Folioso; FOC. Folioso corticado; MI. Microscópicas. Estructura de fijación (EF): CB. Célula basal; DB. Disco basal; MU. Mucílago; ND. No determinado; R. Rizoides. Sustratos animales: M. Molusco; CO. Coral vivo; E. Esponja; B. Balano; SE. Sin especificar. Referencias: 1. Pérez-García (1967); 2. Martinell-Benito (1986); 3. Treviño-Murphy (1986); 4. Dreckmann (1987); 5. Mateo-Cid y Mendoza-González (1991); 6. Mateo-y Mendoza-González (1992); 7. Mendoza-González et al. (1994); 8. León-Tejera et al. (1996); 9. Mendoza-González y Mateo-Cid (1996); 10. Mateo-Cid y Mendoza-González (1997); 11. Ávila-Torres (2006); 12. Rodríguez et al. (2008); 13. PonceMárquez (2010); 14. Mendoza-González et al. (2011); 15. Vázquez-Texocotitla (2013); 16. Sandoval-Coronado (2016); 17. Luna-Barreda (2018); 18. Moncada-García (2018); 19. Este trabajo. *Nuevos registros sólo para Guerrero; **Nuevos registros para el Pacífico tropical mexicano; *** Nuevos registros para el Pacífico de México.

\begin{tabular}{|c|c|c|c|c|c|}
\hline Especies & Estado & GMF & EF & Sustrato & Referencia \\
\hline \multicolumn{6}{|l|}{ Chlorophyta } \\
\hline \multicolumn{6}{|l|}{ Bryopsidales } \\
\hline \multicolumn{6}{|l|}{ Bryopsidaceae } \\
\hline Bryopsis hypnoides J.V. Lamouroux & II & $\mathrm{F}$ & $\mathrm{R}$ & $E$ & 5 \\
\hline Bryopsis pennata J.V. Lamouroux & I & $\mathrm{F}$ & $\mathrm{R}$ & $\mathrm{M}$ & 7 \\
\hline \multicolumn{6}{|l|}{ Caulerpaceae } \\
\hline Caulerpa chemnitzia (Esper) J.V. Lamouroux & VI & $\mathrm{F}$ & $\mathrm{R}$ & $\mathrm{CO}$ & 16 \\
\hline Caulerpa cupressoides (Vahl) C. Agardh & I, III & $\mathrm{F}$ & $\mathrm{R}$ & $\mathrm{M}, \mathrm{CO}$ & 6 \\
\hline Caulerpa racemosa (Forsskål) J. Agardh & I, III & $\mathrm{F}$ & $\mathrm{R}$ & $\mathrm{M}, \mathrm{CO}$ & 6 \\
\hline Caulerpa sertularioides (S.G. Gmelin) M. Howe & $\mathrm{I}, \mathrm{VI}$ & $\mathrm{F}$ & $\mathrm{R}$ & $\mathrm{M}, \mathrm{E}$ & 15 \\
\hline \multicolumn{6}{|l|}{ Codiaceae } \\
\hline Codium setchellii N.L. Gardner & VI & $\mathrm{F}$ & DB & $\mathrm{E}$ & 15 \\
\hline \multicolumn{6}{|l|}{ Derbesiaceae } \\
\hline Derbesia marina (Lyngbye) Solier & VI & $\mathrm{F}$ & $\mathrm{R}$ & $\mathrm{M}, \mathrm{E}$ & $15,19,17$ \\
\hline \multicolumn{6}{|l|}{ Halimedaceae } \\
\hline Halimeda discoidea Decaisne & VI & CA & $\mathrm{R}$ & $\mathrm{CO}$ & 16 \\
\hline \multicolumn{6}{|l|}{ Udoteaceae } \\
\hline Chlorodesmis hildebrandtii A. Gepp \& E.S. Gepp & VI & $\mathrm{F}$ & $\mathrm{R}$ & $\mathrm{CO}$ & 16 \\
\hline \multicolumn{6}{|l|}{ Cladophorales } \\
\hline \multicolumn{6}{|l|}{ Boodleaceae } \\
\hline Boodlea composita (Harvey) F. Brand & I & $\mathrm{F}$ & $\mathrm{R}$ & $\mathrm{M}$ & 7 \\
\hline \multicolumn{6}{|l|}{ Cladophoraceae } \\
\hline Cladophora coelothrix Kützing & III & $\mathrm{F}$ & $\mathrm{R}$ & SE & 14 \\
\hline Cladophora columbiana Collins & VI & $\mathrm{F}$ & $\mathrm{R}$ & M & 19 \\
\hline Cladophora laetevirens Kützing* & VI & $\mathrm{F}$ & $\mathrm{R}$ & M & 19 \\
\hline Cladophora graminea Collins** & VI & $\mathrm{F}$ & $\mathrm{R}$ & $\mathrm{M}$ & 19,7 \\
\hline Cladophora microcladioides Collins & VI & $\mathrm{F}$ & $\mathrm{R}$ & $M, E$ & $15,17,19$ \\
\hline Cladophora sakaii I.A. Abbott & VI & $\mathrm{F}$ & $\mathrm{R}$ & M & 19 \\
\hline Cladophora sericea Kützing & VI & $\mathrm{F}$ & $\mathrm{R}$ & M & 19 \\
\hline Chaetomorpha antennina (Bory) Kützing & VI & $\mathrm{F}$ & $\mathrm{R}$ & $\mathrm{M}$ & 19 \\
\hline Chaetomorpha californica Holden \& Setchell* & VI & $\mathrm{F}$ & $\mathrm{R}$ & M & 19 \\
\hline Chaetomorpha linum (O.F. Müller) Kützing* & VI & $\mathrm{F}$ & $\mathrm{R}$ & M & 19 \\
\hline Chaetomorpha nodosa Kützing*** & VI & $\mathrm{F}$ & $\mathrm{R}$ & M & 19 \\
\hline \multicolumn{6}{|l|}{ Siphonocladaceae } \\
\hline Siphonocladus sp. & VI & $\mathrm{F}$ & $\mathrm{R}$ & M & 19 \\
\hline \multicolumn{6}{|l|}{ Ulotrichales } \\
\hline \multicolumn{6}{|l|}{ Ulotrichaceae } \\
\hline Urospora penicilliformis (Roth) Areschoug*** & VI & $\mathrm{F}$ & $\mathrm{R}$ & M & 19 \\
\hline Urospora wormskioldii (Mertens ex Hornemann) Rosenvinge** & VI & $\mathrm{F}$ & $\mathrm{R}$ & M & 19 \\
\hline Ulothrix flacca (Dillwyn) Thuret* & VI & $\mathrm{F}$ & $\mathrm{R}$ & M & 19 \\
\hline
\end{tabular}


Apéndice: Continuación.

\begin{tabular}{|c|c|c|c|c|c|}
\hline Especies & Estado & GMF & EF & Sustrato & Referencia \\
\hline \multicolumn{6}{|l|}{ Ulvales } \\
\hline \multicolumn{6}{|l|}{ Ulvaceae } \\
\hline Ulva flexuosa Wulfen & VI & FO & DB & M & 19 \\
\hline Ulva intestinalis L. & II, V, VI, VII & FO & DB & M & $2,7,8,19$ \\
\hline \multicolumn{6}{|l|}{ Rhodophyta } \\
\hline \multicolumn{6}{|l|}{ Acrochaetiales } \\
\hline \multicolumn{6}{|l|}{ Acrochaetiaceae } \\
\hline Acrochaetium pacificum Kylin & VI & $\mathrm{F}$ & $\mathrm{R}$ & M & 19 \\
\hline Colaconema coccineum (K.M. Drew) P.W. Gabrielson*** & VI & $\mathrm{F}$ & $\mathrm{R}$ & M & 19 \\
\hline Colaconema daviesii (Dillwyn) Stegenga & VI & $\mathrm{F}$ & $\mathrm{R}$ & $M$ & 19 \\
\hline Colaconema sp. & VI & $\mathrm{F}$ & $\mathrm{R}$ & M & 19 \\
\hline Grania pectinata (Kylin) Athanasiadis & VI & $\mathrm{F}$ & $\mathrm{R}$ & $M$ & 19 \\
\hline
\end{tabular}

\section{Ahnfeltiales}

Ahnfeltiaceae

Ahnfetlia svensonii Taylor

Bangiales

Bangiaceae

Bangia fuscopurpurea (Dillwyn) Lyngbye

VI

Ceramiales

Ceramiaceae

Centroceras clavulatum (C. Agardh) Montagne

Ceramium affine Setchell \& N.L. Gardner

Ceramium camouii E.Y. Dawson

Ceramium caudatum Setchell \& N.L. Gardner

Ceramium dawsonii A.B. Joly

Ceramium hamatispinum E.Y. Dawson

Gayliella flaccida (Harvey ex Kützing) T.O. Cho \& L.J. Mclvor

Delesseriaceae

Dasya sinicola (Setchell \& N.L. Gardner) E.Y. Dawson

Taenioma perpusillum (J. Agardh) J. Agardh

Rhodomelaceae

Chondria repens $\mathrm{B} \emptyset$ rgesen

II

FC

DB

$\mathrm{M}$

Herposiphonia littoralis Hollenberg

Herposiphonia plumula (J. Agardh) Falkenberg

Laurencia clarionensis Setchell \& N.L. Gardner

Laurencia hancockii E.Y. Dawson

Melanothamnus simplex (Hollenberg) Díaz-Tapia \& Maggs

Melanothamnus sp.

Neosiphonia sertularioides (Grateloup) K.W. Nam \& P.J. Kang

Polysiphonia confusa Hollenberg

Polysiphonia pacifica Hollenberg*

Polysiphonia sertularioides (Grateloup) J. Agardh

Polysiphonia subtilissima Montagne

Tayloriella dictyurus(J. Agardh) Kylin

Veleroa subulata E.Y. Dawson

Wrangeliaceae

Anotrichium tenue (C. Agardh) Nägeli

Corallinales

Corallinaceae

Crusticorallina muricata (Foslie) P.W. Gabrielson, Martone, K.R. Hind

\& C.P. Jensen 
Apéndice: Continuación.

\begin{tabular}{|c|c|c|c|c|c|}
\hline Especies & Estado & GMF & EF & Sustrato & Referencia \\
\hline $\begin{array}{l}\text { Chamberlainium decipiens (Foslie) Caragnano, Foetisch, Maneveldt \& } \\
\text { Payri }\end{array}$ & II & C & ND & $\mathrm{M}$ & 5 \\
\hline Jania adhaerens J.V. Lamouroux & I, II & CA & DB & $\mathrm{CO}$ & 6,11 \\
\hline Jania pacifica Areschoug & VI & $\mathrm{CA}$ & DB & B & 1 \\
\hline Jania tenella (Kützing) Grunow & VI & $\mathrm{CA}$ & $\mathrm{R}$ & $\mathrm{CO}$ & 16 \\
\hline Litholepis sonorensis E.Y. Dawson & VI & $\mathrm{C}$ & ND & M & 1 \\
\hline Pneophyllum fragile Kützing & VI & $\mathrm{C}$ & ND & $\mathrm{M}$ & 19 \\
\hline \multicolumn{6}{|l|}{ Hydrolithaceae } \\
\hline Hydrolithon decipiens (Foslie) Adey & I & $\mathrm{C}$ & ND & M & 7 \\
\hline \multicolumn{6}{|l|}{ Lithophyllaceae } \\
\hline Amphiroa beauvoisii J.V.Lamouroux & II, VI & CA & DB & $\mathrm{SE}, \mathrm{CO}$ & 6,16 \\
\hline Amphiroa dimorpha M. Lemoine & II & CA & DB & M & 6 \\
\hline Amphiroa mexicana Taylor & II & $\mathrm{CA}$ & $\mathrm{DB}$ & $\mathrm{M}$ & 6 \\
\hline Amphiroa misakiensis Yendo & VI & $\mathrm{CA}$ & DB & $\mathrm{CO}, \mathrm{E}$ & 15,16 \\
\hline Amphiroa polymorpha M. Lemoine & II & CA & DB & $\mathrm{CO}$ & 6 \\
\hline Amphiroa rigida J.V. Lamouroux & $\mathrm{VI}$ & $\mathrm{CA}$ & DB & $\mathrm{CO}$ & 16 \\
\hline Amphiroa valonioides Yendo & II & $\mathrm{CA}$ & $\mathrm{DB}$ & $\mathrm{CO}$ & 6 \\
\hline Lithophyllum decussatum (J. Ellis \& Solander) Philippi & III & $\mathrm{C}$ & ND & SE & 14 \\
\hline Lithophyllum imitans Foslie & IV & $\mathrm{C}$ & ND & SE & 5 \\
\hline Lithophyllum sp. & VI & $\mathrm{C}$ & ND & M & 19 \\
\hline Tenarea dispar Adey & II & $\mathrm{C}$ & ND & M & 7 \\
\hline \multicolumn{6}{|l|}{ Lithotamniaceae } \\
\hline Lithothamnion sp. & $\mathrm{VI}$ & $\mathrm{C}$ & ND & M & 19 \\
\hline \multicolumn{6}{|l|}{ Porolithaceae } \\
\hline $\begin{array}{l}\text { Dawsoniolithon conicum (E.Y. Dawson) Caragnano, Foetisch, } \\
\text { Maneveldt \& Payri }\end{array}$ & III & $\mathrm{C}$ & ND & SE & 14 \\
\hline \multicolumn{6}{|l|}{ Spongitaceae } \\
\hline Neogoniolithon trichotomum (Heydrich) Setchell \& L.R. Mason & 1 & $\mathrm{C}$ & ND & M & 7 \\
\hline \multicolumn{6}{|l|}{ Erythropeltales } \\
\hline \multicolumn{6}{|l|}{ Erythrotrichiaceae } \\
\hline Erythrotrichia carnea (Dillwyn) J. Agardh & VI & $\mathrm{F}$ & $\mathrm{CB}$ & M & 19 \\
\hline Erythrotrichia porphyroides N.L. Gardner & $\mathrm{VI}$ & $\mathrm{F}$ & $\mathrm{CB}$ & M & 19 \\
\hline Erythrotrichia tetraseriata N.L. Gardner* & $\mathrm{VI}$ & $\mathrm{F}$ & $\mathrm{CB}$ & M & 19 \\
\hline Porphyrostromium pulvinatum West \& Zuccarello** & VI & $\mathrm{F}$ & $\mathrm{CB}$ & M & 19 \\
\hline Sahlingia subintegra (Rosenvinge) Kornmann & $\mathrm{VI}$ & $\mathrm{C}$ & DB & M & 19 \\
\hline \multicolumn{6}{|l|}{ Gelidiales } \\
\hline \multicolumn{6}{|l|}{ Gelidiaceae } \\
\hline Gelidium crinale (Hare ex Turner) Gaillon & I, III, V, VII & FC & $\mathrm{R}$ & M & 13 \\
\hline Gelidium mcnabbianum (E.Y. Dawson) B. Santelices & I, III, VI & FC & $\mathrm{R}$ & $\mathrm{M}, \mathrm{SE}, \mathrm{CO}$ & $12,14,16$ \\
\hline Gelidium microdentatum E.Y. Dawson & II, III, VII & FC & $\mathrm{R}$ & $\mathrm{M}, \mathrm{SE}$ & 10,13 \\
\hline Gelidium pusillum (Stackhouse) Le Jolis & I, II, IV, V, VI & FC & $\mathrm{R}$ & $\mathrm{M}, \mathrm{CO}, \mathrm{E}, \mathrm{B}$ & $2,3,5,6,7,15$ \\
\hline Gelidium sclerophyllum Taylor & I, II, III, V & FC & $\mathrm{R}$ & M & $7,2,13$ \\
\hline \multicolumn{6}{|l|}{ Gelidiellaceae } \\
\hline Parviphycus antipae (Celan) B. Santelices & III & $\mathrm{FC}$ & $\mathrm{R}$ & SE & 14 \\
\hline \multicolumn{6}{|l|}{ Gigartinales } \\
\hline \multicolumn{6}{|l|}{ Cystoclonaceae } \\
\hline Hypnea valentiae (Turner) Montagne & 1 & FC & DB & $\mathrm{M}$ & 7 \\
\hline Hypnea johnstonii Setchell \& N.L. Gardner & $\mathrm{VI}$ & FC & $\mathrm{R}$ & $\mathrm{E}, \mathrm{CO}$ & 16 \\
\hline Hypnea pannosa J. Agardh & $\mathrm{VI}$ & FC & DB & $\mathrm{CO}$ & 15,16 \\
\hline Hypnea spinella (C. Agardh) Kützing & $\mathrm{VI}$ & FC & $\mathrm{R}$ & $\mathrm{CO}$ & 16 \\
\hline
\end{tabular}


Apéndice: Continuación.

\begin{tabular}{|c|c|c|c|c|c|}
\hline Especies & Estado & GMF & EF & Sustrato & Referencia \\
\hline \multicolumn{6}{|l|}{ Phyllophoraceae } \\
\hline Gymnogongrus johnstonii (Setchell \& N.L. Gardner) E.Y. Dawson & II & FC & DB & SE & 6 \\
\hline Gymnogongrus leptophyllus J. Agardh & II & FC & DB & $\mathrm{CO}$ & 6 \\
\hline \multicolumn{6}{|l|}{ Gracilariales } \\
\hline \multicolumn{6}{|l|}{ Gracilariaceae } \\
\hline Gracilaria bursa-pastoris (S.G. Gmelin) P.C. Silva & VII & FC & DB & $\mathrm{M}$ & 8 \\
\hline Gracilaria cerrosiana W.R. Taylor & VII & FC & DB & M & 8 \\
\hline Gracilaria veloroae E.Y. Dawson & VI & FC & DB & $\mathrm{E}$ & 15 \\
\hline Gracilaria tepocensis (E.Y. Dawson) E.Y. Dawson & VII & FC & DB & SE & 8 \\
\hline \multicolumn{6}{|l|}{ Halymeniales } \\
\hline \multicolumn{6}{|l|}{ Halymeniaceae } \\
\hline $\begin{array}{l}\text { Grateloupia clarionensis (Setchell \& N.L. Gardner) S.Kawaguchi \& } \\
\text { H.W. Wang }\end{array}$ & III & FC & DB & SE & 14 \\
\hline Grateloupia versicolor (J. Agardh) J. Agardh & VI & FC & $\mathrm{R}$ & $\mathrm{CO}$ & 16 \\
\hline \multicolumn{6}{|l|}{ Hildenbrandiales } \\
\hline \multicolumn{6}{|l|}{ Hildenbrandiaceae } \\
\hline Hildenbrandia rubra (Sommerfelt) Meneghini & I, IV & $\mathrm{C}$ & ND & M, SE & 7,5 \\
\hline \multicolumn{6}{|l|}{ Peysonneliales } \\
\hline \multicolumn{6}{|l|}{ Peysonneliaceae } \\
\hline Cruoriopsis mexicana E.Y. Dawson & V & $\mathrm{C}$ & ND & $\mathrm{M}$ & 3 \\
\hline Peyssonnelia mexicana E.Y. Dawson & II & $\mathrm{C}$ & ND & M & 6 \\
\hline Peyssonnelia rubra (Greville) J. Agardh & II & $\mathrm{C}$ & ND & M & 14 \\
\hline \multicolumn{6}{|l|}{ Rhodymeniales } \\
\hline \multicolumn{6}{|l|}{ Champiaceae } \\
\hline Champia parvula (C. Agardh) Harvey & $\mathrm{II}, \mathrm{VI}$ & $\mathrm{FC}$ & $\mathrm{R}$ & $\mathrm{CO}$ & 6,16 \\
\hline \multicolumn{6}{|l|}{ Lomentariaceae } \\
\hline Ceratodictyon tenue (Setchell \& N.L. Gardner) J.N. Norris & 1 & FC & DB & M & 7 \\
\hline \multicolumn{6}{|l|}{ Rhodymeniaceae } \\
\hline Rhodymenia sp. & VI & $\mathrm{FC}$ & DB & $\mathrm{M}$ & 19 \\
\hline \multicolumn{6}{|l|}{ Stylonematales } \\
\hline \multicolumn{6}{|l|}{ Stylonemataceae } \\
\hline Chroodactylon ornatum (C. Agardh) Basson & VI & $\mathrm{F}$ & ND & $\mathrm{M}$ & 19 \\
\hline \multicolumn{6}{|l|}{ Ochrophyta } \\
\hline \multicolumn{6}{|l|}{ Dyctiotales } \\
\hline \multicolumn{6}{|l|}{ Dyctiotaceae } \\
\hline Dictyota implexa (Desfontaines) Lamouroux & VII & FOC & $\mathrm{R}$ & $\mathrm{M}$ & 8 \\
\hline \multicolumn{6}{|l|}{ Ectocarpales } \\
\hline \multicolumn{6}{|l|}{ Acinetosporaceae } \\
\hline Hincksia granulosa (Smith) P.C. Silva & V & $\mathrm{F}$ & $\mathrm{R}$ & M & 3 \\
\hline \multicolumn{6}{|l|}{ Chordariaceae } \\
\hline Myrionema strangulans Greville & VI & $\mathrm{C}$ & $\mathrm{R}$ & M & 19 \\
\hline Streblonema sp. & VI & $\mathrm{F}$ & $\mathrm{R}$ & $\mathrm{M}$ & 19 \\
\hline \multicolumn{6}{|l|}{ Ectocarpaceae } \\
\hline Ectocarpus sp. & VI & $\mathrm{F}$ & $\mathrm{R}$ & $\mathrm{M}$ & 19 \\
\hline Ectocarpus siliculosus (Dillwyn) Lyngbye & II & $\mathrm{F}$ & $\mathrm{R}$ & $\mathrm{CO}$ & 6 \\
\hline Feldmannia sp. & VI & $\mathrm{F}$ & $\mathrm{R}$ & $\mathrm{M}$ & 19 \\
\hline Scytosiphonaceae & & & & & \\
\hline Colpomenia ramosa W.R. Taylor & II & FC & $\mathrm{R}$ & SE & 6 \\
\hline Rosenvingea floridana (W.R. Taylor) W.R. Taylor & II & $\mathrm{FC}$ & $\mathrm{R}$ & SE & 6 \\
\hline
\end{tabular}


Apéndice: Continuación.

\begin{tabular}{|c|c|c|c|c|c|}
\hline Especies & Estado & GMF & EF & Sustrato & Referencia \\
\hline \multicolumn{6}{|l|}{ Ralfsiales } \\
\hline \multicolumn{6}{|l|}{ Hapalospongidiaceae } \\
\hline Hapalospongidion gelatinosum Saunders & VI & $\mathrm{C}$ & $\mathrm{R}$ & M & 19 \\
\hline \multicolumn{6}{|l|}{ Neoralfsiaceae } \\
\hline $\begin{array}{l}\text { Neoralfsia hancockii (E.Y. Dawson) D. León-Álvarez \& M.L. Nuñez- } \\
\text { Reséndiz }\end{array}$ & II & $\mathrm{C}$ & $\mathrm{R}$ & M & 6 \\
\hline \multicolumn{6}{|l|}{ Ralfsiaceae } \\
\hline Ralfsia fungiformis (Gunnerus) Setchell \& N.L. Gardner & II & $\mathrm{C}$ & $\mathrm{R}$ & $\mathrm{M}$ & 6 \\
\hline Ralfsia pacifica Hollenberg & IV & C & $\mathrm{R}$ & SE & 5 \\
\hline \multicolumn{6}{|l|}{ Sphacelariales } \\
\hline \multicolumn{6}{|l|}{ Sphacelariaceae } \\
\hline Sphacelaria novae-hollandiae Sonder & VIII & $\mathrm{F}$ & $\mathrm{R}$ & $\mathrm{M}$ & 9 \\
\hline Sphacelaria tribuloides Meneghini & II & $\mathrm{F}$ & $\mathrm{R}$ & $\mathrm{M}$ & 6 \\
\hline \multicolumn{6}{|l|}{ Tilopteridales } \\
\hline \multicolumn{6}{|l|}{ Cutleriaceae } \\
\hline Cutleria hancockii E.Y. Dawson & 1 & FOC & $\mathrm{R}$ & $\mathrm{M}$ & 7 \\
\hline \multicolumn{6}{|l|}{ Cyanobacteria } \\
\hline \multicolumn{6}{|l|}{ Chroococcales } \\
\hline \multicolumn{6}{|l|}{ Microcystaceae } \\
\hline Microcystis sp. & VI & MI & MU & $\mathrm{M}$ & 19 \\
\hline \multicolumn{6}{|l|}{ Nostocales } \\
\hline \multicolumn{6}{|l|}{ Rivulariaceae } \\
\hline Calothrix sp. & VI & MI & MU & M & 16 \\
\hline Microchaete aeruginea Batters & $\mathrm{V}$ & MI & $\mathrm{MU}$ & $\mathrm{M}$ & 4 \\
\hline $\begin{array}{l}\text { Scytonematopsis crustacea (Thuret ex Bornet \& Flahault) Koválik \& } \\
\text { Komárek }\end{array}$ & $\mathrm{V}$ & MI & $\mathrm{MU}$ & M & 4 \\
\hline \multicolumn{6}{|l|}{ Oscillatoriales } \\
\hline \multicolumn{6}{|l|}{ Oscillatoriaceae } \\
\hline Blennothrix lyngbyacea (Kützing ex Gomont) Anagnostidis \& Komárek & $\mathrm{VI}$ & MI & MU & M & 19 \\
\hline Lyngbya majuscula Gomont & VI & MI & $\mathrm{MU}$ & M & 19 \\
\hline Lyngbya sp. & VI & MI & $\mathrm{MU}$ & $\mathrm{M}$ & 19 \\
\hline Porphirosiphon notarisii (Meneghini) Kützing & I & MI & $\mathrm{MU}$ & $\mathrm{M}$ & 7 \\
\hline \multicolumn{6}{|l|}{ Spirulinales } \\
\hline \multicolumn{6}{|l|}{ Spirulinaceae } \\
\hline Spirulina sp. & VI & MI & $\mathrm{MU}$ & $\mathrm{M}$ & 19 \\
\hline
\end{tabular}

